\title{
Phenomenology of nonperturbative charm in the nucleon
}

\author{
T. J. Hobbs ${ }^{1}$, J. T. Londergan ${ }^{1}$, W. Melnitchouk ${ }^{2}$ \\ ${ }^{1}$ Department of Physics and Center for Exploration of Energy and Matter, \\ Indiana University, Bloomington, Indiana 47405, USA \\ ${ }^{2}$ Jefferson Lab, 12000 Jefferson Avenue, Newport News, Virginia 23606, USA
}

(Dated: October 5, 2018)

\begin{abstract}
We perform a comprehensive analysis of the role of nonperturbative (or intrinsic) charm in the nucleon, generated through Fock state expansions of the nucleon wave function involving fivequark virtual states represented by charmed mesons and baryons. We consider contributions from a variety of charmed meson-baryon states and find surprisingly dominant effects from the $\bar{D}^{* 0} \Lambda_{c}^{+}$ configuration. Particular attention is paid to the existence and persistence of high- $x$ structure for intrinsic charm, and the $x$ dependence of the $c-\bar{c}$ asymmetry predicted in meson-baryon models. We discuss how studies of charmed baryons and mesons in hadronic reactions can be used to constrain models, and outline future measurements that could further illuminate the intrinsic charm component of the nucleon.
\end{abstract}




\section{INTRODUCTION}

Charm in the nucleon can be produced in two fundamentally distinct processes. In the first, commonly referred to as "extrinsic charm", charm-anticharm pairs arise through gluon radiation. This perturbative process involving gluon radiation is a significant feature of QCD evolution. Charm arising in this way is concentrated at very low parton momentum fraction $x$, and with increasing $Q^{2}$ one expects to see progressively more charm being produced. Extrinsic charm production ensures essentially identical distributions of charm and anticharm [1].

A second source of charm is called "intrinsic charm." This source of charm arises through nonperturbative fluctuations of the nucleon state to four quark-one antiquark states. Compared with extrinsic charm, intrinsic charm has two rather striking features. First, intrinsic charm tends to be "valence-like," i.e., it is produced at relatively large $x$; second, it is not unusual for intrinsic charm and anticharm to be unequal.

The first calculation of intrinsic charm was made by Brodsky, Hoyer, Peterson and Sakai (BHPS) [2]. They noticed that the first direct measurements of charm production [3-6] were larger than could be accounted for by contemporary calculations of extrinsic charm, and were also more concentrated at relatively large values of Feynman $x_{F}$. BHPS suggested adding in addition to extrinsic charm an "intrinsic" component arising from fluctuations of the nucleon to four quark-one antiquark states. By assuming that the mass of the charm quark in the five-quark configuration was much greater than all other masses and transverse momenta, the distribution of intrinsic charm in $x$ could be computed analytically, with only the overall normalization a free parameter. Although the BHPS ansatz is rather simplistic, it does provide a convenient benchmark for comparing with estimates of charm and anticharm probabilities in more sophisticated treatments. We will review the BHPS formalism in more detail in Sec. II A.

In the intervening years there have also been a number of theoretical calculations of intrinsic charm using various models for production of four quark-one antiquark states [723]. In contrast to the BHPS philosophy, a more dynamical type of model for producing intrinsic quark distributions is based on a two-step approach, in which one considers quarkantiquark production through nonperturbative fluctuations of a proton to a baryon plus meson state. In this fluctuation the heavy quark and antiquark will appear in the resulting 
meson and baryon states. An example of intrinsic charm produced in this way would involve a fluctuation such as $p \rightarrow \Lambda_{c}^{+}+\bar{D}^{0}$, where here the charm quark resides in the $\Lambda_{c}^{+}$baryon (assumed to be a valence $u d c$ state) and the $\bar{c}$ in the $\bar{D}^{0}$ (with valence composition $\bar{c} u$ ). Such models are also referred to as "meson-baryon" models, since one is computing Fock states where a baryon fluctuates to another baryon plus a meson. (These models are also sometimes referred to as "meson-cloud" or "convolution" models, motivated by the SU(2) fluctuation of a proton into a nucleon plus a diffuse "cloud" of nearly massless pions that exist primarily outside a compact baryon core. Of course, the "cloud" analogy is less appropriate for fluctuations to particles containing heavy quarks). A pedagogical review of some of these models was given recently by Pumplin [21].

Since the initial experimental measurements of charm production that inspired the BHPS work, substantial additional experimental data on charm production in high-energy reactions have been collected. Large- $x$ measurements of charm production were carried out by the European Muon Collaboration (EMC) [24] at CERN, and the charm structure function $F_{2}^{c}$ was measured at small $x$ by the H1 [25] and ZEUS [26] Collaborations at HERA. The HERA measurements probe primarily the region where the perturbative extrinsic charm dominates, while the EMC experiment was more sensitive to nonperturbative intrinsic charm.

Despite a number of dedicated theoretical analyses, the magnitude of the intrinsic charm component of the nucleon, which was expected from the early analyses to be of the order of $1 \%$, is still inconclusive. In leptoproduction, the dominant mechanism for perturbative generation of charm is the photon-gluon fusion process. Hoffman and Moore [8] considered $\mathcal{O}\left(\alpha_{s}\right)$ corrections to intrinsic charm distributions, as well as quark and target mass contributions to charm cross sections, finding an intrinsic charm component of order $0.3 \%$ in the proton. Harris, Smith and Vogt [9] reanalyzed the Hoffman-Moore treatment of the EMC data, finding that the EMC charm production data at large energy transfers, $\nu \sim 170 \mathrm{GeV}$, required an intrinsic charm component normalized to $0.86 \pm 0.60 \%$. A subsequent study by Steffens et al. [16] used the BHPS and meson-baryon intrinsic charm models, together with an interpolating scheme, to map smoothly onto massless QCD evolution at large $Q^{2}$ and photon-gluon fusion at small $Q^{2}$. While the analysis found it difficult to simultaneously fit the entire data set in terms of a single intrinsic charm scenario, it did indicate a slight preference for intrinsic charm in a meson-baryon model at a level of about $0.4 \%$, although the evidence was not conclusive. 
The most detailed study of intrinsic charm within a global QCD analysis of parton distribution functions (PDFs) was made by Pumplin, Lai and Tung [22], using models for intrinsic charm from Ref. [21], and recently updated by Dulat et al. [23] to include next-to-next-to leading (NNLO) order $\alpha_{s}$ corrections. Depending on the intrinsic charm model used, they found that the high-energy data could accommodate up to about $2 \%$ of intrinsic charm without serious disagreement with the high-energy data. On the other hand, the analyses of Refs. [22, 23] did not include the original EMC data [24] that suggested an enhancement in $F_{2}^{c}$ at large $x$. In fact, since there is some tension between the EMC and HERA data at small values of $x$, typically global PDF analyses omit the EMC data and fit the more extensive HERA data in terms of perturbative contributions alone [27, 28].

Clearly, additional data are needed for a more definitive determination of the role of intrinsic charm in the nucleon, especially at large $x$, and several new charm production experiments that have been proposed recently may shed light on the dynamical origin of nonperturbative charm. Studies of $D N$ and $J / \psi N$ interactions, charmed baryon spectroscopy and charmed nuclei will be possible, for example, at the J-PARC facility in Japan [29], with its primary proton and secondary pion beams, while proton and antiproton beams can be used to study open charm production at GSI-FAIR in Germany [30]. Near threshold $J / \psi$ photoproduction at Jefferson Lab, following its $12 \mathrm{GeV}$ upgrade, would allow gluon generalized parton distributions to be explored, in addition to the $A$ dependence of the $J / \psi$-nucleon cross section [31, 32]. For the proposed AFTER@CERN fixed-target facility [33], charmed meson production has been suggested as a means of tagging the gluon distribution at large $x$, which requires understanding of competing contributions from charm quarks at similar kinematics. Finally, a future Electron-Ion Collider [34-36] would provide opportunities to access charm in the nucleon in the large- $x$ region.

Anticipating these future experimental efforts, and in view of the somewhat conflicting results from the previous phenomenological studies, it is therefore timely to revisit the problem of intrinsic charm using the latest available theoretical techniques and models for calculating $F_{2}^{c}$. In this paper we undertake a comprehensive and critical analysis of nonperturbative models of charm in the nucleon, with the aim of obtaining a more robust understanding of both the magnitude and shape of the intrinsic charm distribution. We begin by reviewing in Sec. II the simple five-quark models of the nucleon introduced by BHPS [2] and Pumplin [21]. In Sec. III, we discuss the more dynamical meson-baryon models for heavy quark 
generation, which are constrained by coupled-channel analyses of baryon-baryon scattering [37-40]. These were initially used to describe nucleon-nucleon scattering, then were applied to the strange sector, and have since been extended to the regime of charm. We summarize the assumptions made in the extensions into the strange and charmed sectors, and outline the experimental data that fix the coupling constants and cutoff parameters for such potentials. We compare the meson-baryon model calculations with hadronic data on production of charmed baryons from the R608 Collaboration at the ISR [41], which measured inclusive $\Lambda_{c}$ production in $p p$ collisions. We also examine charge asymmetries in $\Lambda_{c}$ production from proton beams which has been measured recently by the SELEX Collaboration [42].

The basic ingredients of the meson-baryon models, namely, the proton $\rightarrow$ meson + baryon splitting functions and the charm quark distributions in the charmed $D$ mesons and baryons, are derived in Secs. IV and V, respectively. The resulting convolutions of these distributions are presented in Sec. VI, where we compare various prescriptions for the regularization of the momentum integrals, and different approximations for the distributions in the charmed hadrons. We also confront the models with data on the charm structure function $F_{2}^{c}(x)$, in particular with the EMC data [24] which measured charm at large $x$, and in addition the very precise HERA data from the H1 and ZEUS Collaborations [25, 26], which is sensitive to charm in the low- $x$ region. Finally, in Sec. VII we summarize our results, and discuss possibilities for the measurements of charmed observables at current and future facilities. Additional technical details of the derivations of the meson-baryon splitting functions and the charm quark distributions in charmed hadrons are given in Appendix A and $\mathrm{B}$, respectively, and simple parametrizations of the calculated $c$ and $\bar{c}$ distributions in the nucleon are provided in Appendix C.

\section{FIVE-QUARK MODELS OF NUCLEON STRUCTURE}

In this section we review models of intrinsic charm based on particular five-quark Fock state components of the nucleon wave function. We will focus on models that describe the

process by which a nucleon initially containing three light valence quarks transitions to a four quark plus one antiquark state containing a charm-anticharm quark pair. 


\section{A. The BHPS model}

The simplest model for producing intrinsic charm was proposed over 30 years ago by BHPS [2]. In the infinite momentum frame (IMF) the transition probability for a proton with mass $M$ to make a transition $p \rightarrow u u d c \bar{c}$ (or to a five-quark state containing any heavy quark pair) involves an energy denominator that can be expressed in terms of the masses $m_{i}$ and momentum fractions $x_{i}$ of the constituents,

$$
P(p \rightarrow u u d c \bar{c}) \sim\left[M^{2}-\sum_{i=1}^{5} \frac{k_{\perp i}^{2}+m_{i}^{2}}{x_{i}}\right]^{-2} .
$$

Here, $k_{\perp i}$ is the transverse momentum of quark $i$, and the heavy quarks in the $c \bar{c}$ pair are assigned indices 4 and 5 .

For simplicity, the BHPS calculation assumed a point coupling for the $c \bar{c}$ production vertex, and neglected the effect of transverse momentum in the five-quark transition amplitudes. With the additional assumption that the charm mass is much greater than the nucleon and light quark masses, the probability for producing a single charm quark can be derived analytically,

$$
P\left(x_{5}\right)=\frac{N x_{5}^{2}}{2}\left[\frac{\left(1-x_{5}\right)}{3}\left(1+10 x_{5}+x_{5}^{2}\right)+2 x_{5}\left(1+x_{5}\right) \ln \left(x_{5}\right)\right],
$$

with the normalization $N$ fixed by the overall charm quark probability in the proton.

Using the analytic expression (2) one obtains a "valence-like" charm quark distribution that is significant for $0.1 \leq x_{5} \leq 0.5$. The valence-like shape arises from the structure of the energy denominator in Eq. (1), which for large quark masses $m_{i}$ favors configurations with large momentum fractions $x_{i}$. This feature is common to all similar five-quark models and invariably results in a valence-like heavy quark distribution.

It is also possible to compute the charm quark probability numerically without assuming that the charm quark mass is much greater than all other masses. With realistic masses, the charm quark distribution turns out to be similar to the analytic form of Eq. (2). Note also that since the charm and anticharm probabilities enter Eq. (1) symmetrically, the charm production mechanism in this model will produce equal probabilities for $c$ and $\bar{c}$. Although the BHPS model is rather simplistic, it nevertheless provides a useful reference point of comparison to test intrinsic charm and anticharm distributions obtained from other prescriptions. 


\section{B. Scalar five-quark model}

In a detailed study of intrinsic heavy quark probabilities, Pumplin [21] considered a series of models for the Fock space wave function on the light-front for a proton to make a transition to a four quark plus one antiquark system, with the heavy $q \bar{q}$ pair composed of either charm or bottom quarks. A simplified case was studied where a point scalar particle of mass $m_{0}$ couples with strength $g$ to $N$ scalar particles with masses $m_{1}, m_{2}, \ldots, m_{N}$. The light-front Fock space probability density $d P$ for such a process then takes the form [21]

$$
d P=\frac{g^{2}}{\left(16 \pi^{2}\right)^{N-1}(N-2) !} \prod_{j=1}^{N} d x_{j} \delta\left(1-\sum_{j=1}^{N} x_{j}\right) \int_{s_{0}}^{\infty} d s \frac{\left(s-s_{0}\right)^{N-2}}{\left(s-m_{0}^{2}\right)^{2}}|F(s)|^{2},
$$

where $s_{0}=\sum_{j=1}^{N} m_{j}^{2} / x_{j}$, and the form factor $F(s)$ serves to suppress contributions from high-mass states. If one neglects the effects of transverse momentum and the factors of $1 / x_{j}$ in Eq. (3), and assumes a point form factor $F(s)=1$, then in the limit that the charm mass is much larger than all other masses one recovers the distribution in the BHPS model [2].

To incorporate the effects of the finite size of the nucleon, Pumplin considered both an exponential form factor,

$$
|F(s)|^{2}=\exp \left[-\left(s-m_{0}^{2}\right) / \Lambda^{2}\right]
$$

and a power-law suppression factor,

$$
|F(s)|^{2}=\frac{1}{\left(s+\Lambda^{2}\right)^{n}}
$$

with $\Lambda$ a cutoff mass regulator. Fixing the overall normalization to be a constant, the resulting shape of the charm quark momentum distribution with the power-law suppression, for a reasonable choice of $n=4$, turns out to be softer than the BHPS prediction for a range of cutoffs, $\Lambda=2-10 \mathrm{GeV}$ [21]. For the exponential suppression, the shape depends somewhat more strongly on the cutoff parameter, with the distribution being harder than the BHPS result for smaller $\Lambda$ values and softer for larger $\Lambda$. All of the resulting charm distributions are valence-like, however, with significant tails even beyond $x \approx 0.4$.

While the simple five-quark models give some qualitative insights into the possible generation of intrinsic charm at large $x$, they retain a high degree of dependence on the model parameters, whose connection with the underlying QCD theory is not clear. Furthermore, it is also not obvious how one could constrain the parameters phenomenologically by comparing deep-inelastic scattering with other observables, for instance. In the next section we 
discuss an alternative approach which may offer greater promise for relating intrinsic charm distributions to inputs determined from independent reactions.

\section{MESON-BARYON MODELS FOR INTRINSIC CHARM}

A hybrid class of intrinsic charm models that involves both quark and hadron degrees of freedom, and makes some unique and testable predictions for the $c$ and $\bar{c}$ distributions in the nucleon, are meson-baryon models. Such models attempt to quantify the fluctuations of the nucleon to states with a virtual meson $M$ plus baryon $B$,

$$
|N\rangle=\sqrt{Z_{2}}|N\rangle_{0}+\sum_{M, B} \int d y d^{2} \boldsymbol{k}_{\perp} \phi_{M B}\left(y, k_{\perp}^{2}\right)\left|M\left(y, \boldsymbol{k}_{\perp}\right) ; B\left(1-y,-\boldsymbol{k}_{\perp}\right)\right\rangle,
$$

where $|N\rangle_{0}$ is the "bare", three-quark nucleon state, and $Z_{2}$ is the wave function renormalization. The function $\phi_{M B}\left(y, k_{\perp}^{2}\right)$ gives the probability amplitude for the physical nucleon to be in a state consisting of a virtual meson $M$ with longitudinal momentum fraction $y$ and transverse momentum $\boldsymbol{k}_{\perp}$, and a baryon $B$ with longitudinal momentum fraction $1-y$ and transverse momentum $-\boldsymbol{k}_{\perp}$. The total invariant mass squared of the meson-baryon system $s_{M B}$ can be written in the IMF as

$$
s_{M B}\left(y, k_{\perp}^{2}\right)=\frac{k_{\perp}^{2}+m_{M}^{2}}{y}+\frac{k_{\perp}^{2}+M_{B}^{2}}{1-y},
$$

where $m_{M}$ and $M_{B}$ are the meson and baryon masses, respectively. If the meson-baryon terms include states containing charm quarks, the resulting probability distributions for anticharm and charm quarks in the nucleon can be written in the form of convolutions,

$$
\begin{aligned}
& \bar{c}(x)=\sum_{M, B}\left[\int_{x}^{1} \frac{d y}{y} f_{M B}(y) \bar{c}_{M}\left(\frac{x}{y}\right)+\int_{x}^{1} \frac{d \bar{y}}{\bar{y}} f_{B M}(\bar{y}) \bar{c}_{B}\left(\frac{x}{\bar{y}}\right)\right], \\
& c(x)=\sum_{B, M}\left[\int_{x}^{1} \frac{d \bar{y}}{\bar{y}} f_{B M}(\bar{y}) c_{B}\left(\frac{x}{\bar{y}}\right)+\int_{x}^{1} \frac{d y}{y} f_{M B}(y) c_{M}\left(\frac{x}{y}\right)\right],
\end{aligned}
$$

where $\bar{y} \equiv 1-y$, and for ease of notation we have omitted the dependence of the distributions on the scale $Q^{2}$.

In analogy with the quark-gluon splitting functions of perturbative QCD, in Eqs. (8) $f_{M B}(y)$ represents the splitting function for a nucleon to fluctuate to meson $M$ with fraction $y$ of the proton's momentum, and a spectator baryon $B$. The charm and anticharm distributions in the baryon $B$ are denoted by $c_{B}(z)$ and $\bar{c}_{B}(z)$, respectively, and carry a fraction 
$z=x / \bar{y}$ of the baryon's momentum. Similarly, $f_{B M}(\bar{y})$ represents the splitting function for a nucleon fluctuating into a baryon $B$ with fraction $\bar{y}$ of the proton's momentum, with a spectator meson $M$. The quark distributions inside the meson $M$ are denoted by $c_{M}(z)$ and $\bar{c}_{M}(z)$, respectively. If the charm quark resides exclusively in the baryon, with the anticharm in the meson, Eqs. (8) simplify further since

$$
c_{M}(x) \rightarrow 0, \quad \bar{c}_{B}(x) \rightarrow 0 .
$$

The splitting functions in Eqs. (8) are related to the probability amplitudes $\phi_{M B}$ by

$$
f_{M B}(y)=\int_{0}^{\infty} d^{2} \boldsymbol{k}_{\perp}\left|\phi_{M B}\left(y, k_{\perp}^{2}\right)\right|^{2}=f_{B M}(\bar{y}),
$$

where the reciprocity relation in the second equality arises from the conservation of threemomentum at the $M B N$ vertex [43-47]. It can be shown to be satisfied explicitly in the infinite momentum frame (or on the light-front), but is violated in covariant calculations [44-48] in the presence of $M B N$ form factors (or other ultraviolet regulators) which do not exhibit the $y \leftrightarrow \bar{y}$ symmetry of the amplitudes $\phi_{M B}$ [49].

The convolution equations (8) allow the symmetries of the splitting functions to be represented in terms of moments of the parton distributions $C^{(n)}$ and $\bar{C}^{(n)}$, defined as

$$
\begin{aligned}
& \bar{C}^{(n)}=\int_{0}^{1} d x x^{n} \bar{c}(x)=\sum_{M, B} \mathcal{F}_{M B}^{(n)} \bar{C}_{M}^{(n)}, \\
& C^{(n)}=\int_{0}^{1} d x x^{n} c(x)=\sum_{B, M} \mathcal{F}_{B M}^{(n)} C_{B}^{(n)},
\end{aligned}
$$

where

$$
\begin{aligned}
\mathcal{F}_{M B}^{(n)} & =\int_{0}^{1} d y y^{n} f_{M B}(y), \\
\mathcal{F}_{B M}^{(n)} & =\int_{0}^{1} d \bar{y} \bar{y}^{n} f_{B M}(\bar{y}),
\end{aligned}
$$

are the $n$-th moments of the splitting functions. The corresponding moments of the $\bar{c}$ and $c$ distributions in the meson $M$ and baryon $B$ are denoted by $\bar{C}_{M}^{(n)}$ and $C_{B}^{(n)}$, respectively. In particular, the lowest moment of the splitting functions gives the average multiplicity of mesons $M$ and baryons $B$,

$$
\langle n\rangle_{M B} \equiv \mathcal{F}_{M B}^{(0)}=\mathcal{F}_{B M}^{(0)},
$$


which reflects global charge conservation, while conservation of momentum implies that the momentum fractions $\langle y\rangle_{M B} \equiv \mathcal{F}_{M B}^{(1)}$ and $\langle y\rangle_{B M} \equiv \mathcal{F}_{B M}^{(1)}$ are related by

$$
\langle y\rangle_{B M}+\langle y\rangle_{M B}=\langle n\rangle_{M B} .
$$

In contrast to the five-quark models discussed in Sec. II, in which the $x$ dependence of the $c$ and $\bar{c}$ distributions was identical, in the MBM the distributions of heavy quarks and antiquarks in the nucleon are generally expected to be different. Indeed, since the $c$ in the baryon and $\bar{c}$ in the meson reside in rather different local environments, an asymmetry $c(x) \neq \bar{c}(x)$ is almost unavoidable. Of course, since the proton has no net charm, the lowest moments of $c$ and $\bar{c}$ must cancel; however, all higher moments will be nonzero,

$$
C^{(0)}-\bar{C}^{(0)}=0, \quad C^{(n)}-\bar{C}^{(n)} \neq 0 \quad(n \geq 1) .
$$

Whenever Eq. (13) is satisfied, this will guarantee that the proton has no net charm.

Because quarks and antiquarks possess opposite intrinsic parities, parity conservation will require that the quark wave functions respect overall parity conservation. For example if the initial proton state is treated as three constituent (uud) quarks in $S$-wave orbitals and a $c \bar{c}$ pair is added, then if one of the charm quarks is placed in an $S$ state the other needs to be in an odd-parity state. In the MBM, this behavior is accommodated provided one uses physical vertices that correctly account for the spin degrees of freedom of the relevant fields. Models that treat quarks as scalar point-like particles, for example, will generally not satisfy these constraints [21, 22].

In the present analysis, we consider various meson-baryon states containing charm quarks that could contribute to the intrinsic charm in the proton, as summarized in Table I. These include the $\mathrm{SU}(4)$ octet isoscalar $\Lambda_{c}$ and isovector $\Sigma_{c}$ baryons, and the decuplet $\Sigma_{c}^{*}$, while for the mesons, the pseudoscalar $D$ and vector $D^{*}$ mesons are included. In addition, the state involving a proton fluctuation to $p+J / \psi$, where both the $c$ and $\bar{c}$ reside in the $J / \psi$, was considered in Ref. [21]. Although this has a combined mass which is actually lower than all the other charmed meson-baryon configurations, its contribution should be strongly suppressed by the OZI rule.

In the following section the splitting functions $f_{M B}(y)$ for the various configurations in Table I will be presented, with details of the derivations given in Appendix A. To constrain the model parameters in the calculations, namely, the hadronic couplings and form fac- 
TABLE I: Lowest mass meson-baryon Fock states of the proton containing charm and anticharm quarks. For each state the isospin $I$, spin $J$ and parity $P$ are listed for the meson and baryon, together with the masses.

\begin{tabular}{lccc}
\hline \hline Baryon & $I\left(J^{P}\right)$ & Meson & $I\left(J^{P}\right)$ \\
\hline$p(938)$ & ${\frac{1}{2}\left(\frac{1}{2}^{+}\right)} J / \psi(3097)$ & $0\left(1^{-}\right)$ \\
$\Lambda_{c}^{+}(2286)$ & $0\left(\frac{1}{2}^{+}\right)$ & $\bar{D}^{0}(1865)$ & $\frac{1}{2}\left(0^{-}\right)$ \\
& & $\bar{D}^{* 0}(2007)$ & $\frac{1}{2}\left(1^{-}\right)$ \\
$\Sigma_{c}^{+}(2455)$ & $1{\left(\frac{1}{2}^{+}\right)}^{+}$ & $\bar{D}^{0}(1865)$ & $\frac{1}{2}\left(0^{-}\right)$ \\
& & $\bar{D}^{* 0}(2007)$ & $\frac{1}{2}\left(1^{-}\right)$ \\
$\Sigma_{c}^{++}(2455)$ & $1\left(\frac{1}{2}^{+}\right)$ & $D^{-}(1870)$ & $\frac{1}{2}\left(0^{-}\right)$ \\
& & $D^{*-}(2010)$ & $\frac{1}{2}\left(1^{-}\right)$ \\
$\Sigma_{c}^{*+}(2520)$ & $1\left(\frac{3}{2}^{+}\right)$ & $\bar{D}^{0}(1865)$ & $\frac{1}{2}\left(0^{-}\right)$ \\
& & $\bar{D}^{* 0}(2007)$ & $\frac{1}{2}\left(1^{-}\right)$ \\
$\Sigma_{c}^{*++}(2520)$ & $1\left(\frac{3}{2}^{+}\right)$ & $D^{-}(1870)$ & $\frac{1}{2}\left(0^{-}\right)$ \\
& & $D^{*-}(2010)$ & $\frac{1}{2}\left(1^{-}\right)$ \\
\hline
\end{tabular}

tor cutoffs, we use phenomenological input from $D N$ and $\bar{D} N$ scattering analyses [37-40], together with inclusive charmed baryon production data in $p p$ collisions.

\section{CHARMED MESON-BARYON SPLITTING FUNCTIONS}

For a given meson-baryon state $M B$, the splitting function can be evaluated as in Eq. (10) in terms of an integral over the transverse momentum of the square of the probability amplitude $\phi_{B M}\left(y, k_{\perp}^{2}\right)$ which was defined in Eq. (6). To compute the probability amplitudes we use time-ordered perturbation theory, characterized by a particular choice of forwardmoving kinematics in the IMF. Details of the framework and complete derivations follow in Appendix A.

We consider splitting functions for $p \rightarrow M B$ fluctuations for the spin transitions $\frac{1}{2} \rightarrow \mathbf{0} \bigoplus \frac{1}{2}, \mathbf{1} \bigoplus \frac{1}{2}, \mathbf{0} \bigoplus \frac{3}{2}$, and $\mathbf{1} \bigoplus \frac{3}{2}$, with the specific states listed in Table I and illustrated in Fig. 1 (with the exception of the $p J / \psi$ state). Couplings to these states are then determined from the lowest-order effective hadronic Lagrangian for each transition, 


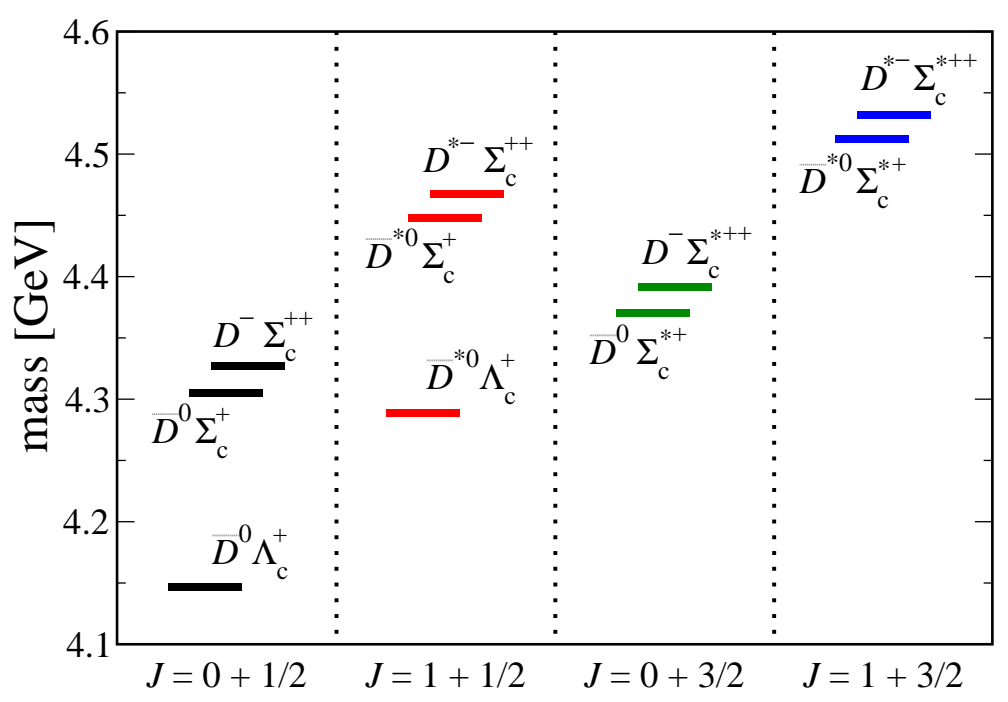

FIG. 1: (color online) Spin configurations $(J=$ meson + baryon spin) and masses for the spectrum of charmed hadron configurations included in the present MBM calculation.

given in Appendix A.

In the framework of the MBM, the splitting function for the fluctuation of the proton to a spin-0 charmed meson $D=\bar{D}^{0}$ or $D^{-}$and a spin-1/2 charmed baryon $B=\Lambda_{c}^{+}, \Sigma_{c}^{+}$or $\Sigma_{c}^{++}$is given by

$$
f_{D B}(y)=T_{B} \frac{g^{2}}{16 \pi^{2}} \int \frac{d k_{\perp}^{2}}{y(1-y)} \frac{|F(s)|^{2}}{\left(s-M^{2}\right)^{2}}\left[\frac{k_{\perp}^{2}+\left(M_{B}-(1-y) M\right)^{2}}{1-y}\right],
$$

where for ease of notation we have used for the coupling constant $g \rightarrow g_{D B N}$ and for the $D B$ invariant mass $s \rightarrow s_{D B}$. The isospin transition factor $T_{B}$ is given by

$$
T_{B}=1+\delta_{t_{B},+1}
$$

where the third component of the isospin of the charmed baryon is $t_{B}=0$ for $B=\Lambda_{c}^{+}$ and $\Sigma_{c}^{+}$, and $t_{B}=+1$ for $B=\Sigma_{c}^{++}$. The states described by the splitting function $f_{D B}(y)$ include the lowest-mass configuration $\bar{D}^{0} \Lambda_{c}^{+}$, as well as the isovector charmed baryon states $\bar{D}^{0} \Sigma_{c}^{+}$and $D^{-} \Sigma_{c}^{++}$.

At large transverse momenta, the invariant mass $s \sim k_{\perp}^{2}$, making the integral in Eq. (16) logarithmically divergent. A simple way to regulate this divergence is with a form factor $F(s)$, which acts to suppress the ultraviolet contributions. A convenient way to parametrize this regularizing form factor is using an exponential function of $s$,

$$
F(s)=\exp \left[-\left(s-M^{2}\right) / \Lambda^{2}\right]
$$


which has the merit of possessing simple normalization properties on-shell, although multipole, or power-law, functional forms as in Eq. (5) would also suffice. The splitting functions also depend upon the magnitude of the coupling $g$ to each meson-baryon state, which we take from baryon-baryon scattering models extended to the charm sector. For simplicity, we assume a universal exponential form factor for all couplings, where the scale factor $\Lambda$ for that form factor is varied to fit charmed baryon production in hadronic interactions, as discussed below. (Note, however, that the exponential form is used to define the form factor $F(s)$ in Eq. (18), in contrast to the square of the form factor, as in Eq. (4); the two can of course be related by a simple rescaling of the cutoff mass $\Lambda^{2} \rightarrow 2 \Lambda^{2}$ ).

For the dissociation of the proton to a charmed vector meson $D^{*}=\bar{D}^{* 0}$ or $D^{*-}$ and spin-1/2 charmed baryon, the corresponding splitting function is given by a sum of vector $\left(G_{v}\right)$, tensor $\left(G_{t}\right)$ and vector-tensor interference $\left(G_{v t}\right)$ terms,

$$
\begin{aligned}
f_{D^{*} B}(y)= & T_{B} \frac{1}{16 \pi^{2}} \int \frac{d k_{\perp}^{2}}{y(1-y)} \frac{|F(s)|^{2}}{\left(s-M^{2}\right)^{2}} \\
& \times\left[g^{2} G_{v}\left(y, k_{\perp}^{2}\right)+\frac{g f}{M} G_{v t}\left(y, k_{\perp}^{2}\right)+\frac{f^{2}}{M^{2}} G_{t}\left(y, k_{\perp}^{2}\right)\right],
\end{aligned}
$$

where

$$
\begin{aligned}
G_{v}\left(y, k_{\perp}\right)= & -6 M M_{B}+\frac{4(P \cdot k)(p \cdot k)}{m_{D}^{2}}+2 P \cdot p \\
G_{v t}\left(y, k_{\perp}\right)= & 4\left(M+M_{B}\right)\left(P \cdot p-M M_{B}\right) \\
& -\frac{2}{m_{D}^{2}}\left[M_{B}(P \cdot k)^{2}-\left(M+M_{B}\right)(P \cdot k)(p \cdot k)+M(p \cdot k)^{2}\right] \\
G_{t}\left(y, k_{\perp}\right)= & -(P \cdot p)^{2}+\left(M+M_{B}\right)^{2} P \cdot p-M M_{B}\left(M^{2}+M_{B}^{2}+M M_{B}\right) \\
& +\frac{1}{2 m_{D}^{2}}\left[\left(P \cdot p-M M_{B}\right)[(P-p) \cdot k]^{2}-2\left(M_{B}^{2} P \cdot k-M^{2} p \cdot k\right)[(P-p) \cdot k]\right. \\
& \left.+2(P \cdot k)(p \cdot k)\left(2 P \cdot k-M_{B}^{2}-M^{2}\right)\right]
\end{aligned}
$$

where $p$ is the four-momentum of the baryon, and the inner products $P \cdot p, P \cdot k$ and $p \cdot k$ can be computed from Eq. (A3). The splitting function $f_{D^{*} B}(y)$ describes transitions to the states $\bar{D}^{* 0} \Lambda_{c}^{+}, \bar{D}^{* 0} \Sigma_{c}^{+}$and $D^{*-} \Sigma_{c}^{++}$, and the isospin transition factor $T_{B}$ is as in Eq. (17). Again, for ease of notation, we have used the shorthand notation for the couplings $g \rightarrow g_{D^{*} B N}$ and $f \rightarrow f_{D^{*} B N}$, with $s \rightarrow s_{D^{*} B}$.

For completeness, we also include fluctuations to spin-3/2 charmed baryons $B^{*}=\Sigma_{c}^{*+}$ and $\Sigma_{c}^{*++}$. For the dissociations of a proton to states with a spin-0 $D$ meson, $\bar{D}^{0} \Sigma_{c}^{*+}$ and 
$D^{-} \sum_{c}^{*++}$, the splitting function is given by

$$
\begin{aligned}
f_{D B^{*}}(y)= & T_{B^{*}} \frac{g^{2}}{16 \pi^{2}} \int \frac{d k_{\perp}^{2}}{y(1-y)} \frac{|F(s)|^{2}}{\left(s-M^{2}\right)^{2}} \\
& \times \frac{\left[k_{\perp}^{2}+\left(M_{B^{*}}-(1-y) M\right)^{2}\right]\left[k_{\perp}^{2}+\left(M_{B^{*}}+(1-y) M\right)^{2}\right]^{2}}{6 M_{B^{*}}^{2}(1-y)^{3}},
\end{aligned}
$$

with $g \rightarrow g_{D B^{*} N}$ and $s \rightarrow s_{D B^{*}}$. The isospin transition factor $T_{B^{*}}$ here is similar to that in Eq. (17), but with the third component of the charmed baryon isospin $t_{B^{*}}=0$ for $B^{*}=\Sigma_{c}^{*+}$ and $t_{B^{*}}=+1$ for $B^{*}=\Sigma_{c}^{*++}$.

Finally, for the fluctuations to states with $D^{*}$ mesons and spin-3/2 baryons $B^{*}$, the splitting function is

$$
\begin{aligned}
f_{D^{*} B^{*}}(y)= & T_{B^{*}} \frac{g^{2}}{m_{D^{*}}^{2} 16 \pi^{2}} \int \frac{d k_{\perp}^{2}}{y(1-y)} \frac{|F(s)|^{2}}{\left(s-M^{2}\right)^{2}} \\
& -\left[\frac{4 M M_{B^{*}}}{3}\left(2 M_{B^{*}}^{2}+M M_{B^{*}}+2 M^{2}\right)-\frac{4 M M_{B^{*}}}{3 m_{D^{*}}^{2}}((P-p) \cdot k)^{2}\right. \\
& -\frac{4}{3 m_{D^{*}}^{2}}\left(M_{B^{*}}^{2}(P \cdot k)^{2}+M^{2}(p \cdot k)^{2}\right)+\frac{4 P \cdot p}{3}\left(2 M_{B^{*}}^{2}+4 M M_{B^{*}}+M^{2}\right) \\
& \left.+\frac{4 P \cdot p}{3 m_{D^{*}}^{2}}(p \cdot k)^{2}\left(1-\frac{M^{2}}{M_{B^{*}}^{2}}\right)-4(P \cdot p)^{2}\left(1-\frac{2(P \cdot k)(p \cdot k)}{3 m_{D^{*}}^{2} M_{B^{*}}^{2}}-\frac{P \cdot p}{3 M_{B^{*}}^{2}}\right)\right],
\end{aligned}
$$

with $g \rightarrow g_{D^{*} B^{*} N}, s \rightarrow s_{D^{*} B^{*}}$, and the inner products in Eq. (22) obtained from Eq. (A3) with the replacements $D \rightarrow D^{*}$ and $B_{c} \rightarrow \Sigma_{c}^{*}$. The details of the derivations of all of the functions $f_{D B}(y), f_{D^{*} B}(y), f_{D B^{*}}(y)$ and $f_{D^{*} B^{*}}(y)$ can be found in Appendix A.

\section{A. Constraints from inclusive charmed hadron production}

To calculate the contributions of the various charmed mesons and baryons listed in Table I requires the couplings of these states to the proton. In this analysis we take the coupling constants from boson-exchange models that were originally applied to pion-nucleon interactions [50], and later generalized to $K N$ scattering [51]. In the extension to the strange sector, the relevant couplings are taken from non-strange analyses with $\mathrm{SU}(3)$ arguments used to incorporate the corresponding strange particles. The off-shell behavior of the amplitudes is typically regulated by a multipole form factor of the type

$$
F(t)=\left(\frac{\Lambda^{2}+m_{M}^{2}}{\Lambda^{2}-t}\right)^{n},
$$


TABLE II: Charm-sector coupling constants, deduced from $D N$ and $\bar{D} N$ scattering analyses [40] for the spin-1/2 charmed baryons $\Lambda_{c}$ and $\Sigma_{c}$, and by extending the SU(3) sector analysis of Ref. [52] for the spin-3/2 $\Sigma_{c}^{*}$ states.

\begin{tabular}{lcc}
\hline \hline Vertex & $g_{M B N} / \sqrt{4 \pi}$ & $f_{M B N} / \sqrt{4 \pi}$ \\
\hline$p \rightarrow \bar{D}^{0} \Lambda_{c}^{+}$ & 3.943 & - \\
$p \rightarrow \bar{D}^{* 0} \Lambda_{c}^{+}$ & 1.590 & 5.183 \\
$p \rightarrow \bar{D}^{0} \Sigma_{c}^{+}, D^{-} \Sigma_{c}^{++}$ & 0.759 & - \\
$p \rightarrow \bar{D}^{* 0} \Sigma_{c}^{+}, D^{*-} \Sigma_{c}^{++}$ & 0.918 & -2.222 \\
$p \rightarrow \bar{D}^{0} \Sigma_{c}^{*+}, D^{-} \Sigma_{c}^{*++}$ & -0.193 & - \\
$p \rightarrow \bar{D}^{* 0} \Sigma_{c}^{*+}, D^{*-} \Sigma_{c}^{*++}$ & -1.846 & - \\
\hline
\end{tabular}

where $t$ is the usual Mandelstam variable for the squared momentum transfer of the exchanged meson with mass $m_{M}$. A monopole form factor $(n=1)$ is generally used for lowspin states, while for higher-spin states a dipole form factor $(n=2)$ is typically employed to damp the higher powers of momentum that enter into the transition amplitudes.

The extension of meson-baryon couplings from the non-strange to the strange sector has generally been quite successful phenomenologically. Continuing this program further to the charm sector, Haidenbauer et al. [38-40] used SU(4) symmetry arguments to describe exclusive charmed hadron production in $\bar{D} N$ and $D N$ scattering within a one-boson-exchange framework. We fix the couplings for the spin-1/2 charmed baryons $\Lambda_{c}$ and $\Sigma_{c}$ to those found in Ref. [40], as summarized in Table II. For the couplings to spin-3/2 states $\Sigma_{c}^{*}$, we take the couplings from those obtained for the analogous strange states by Holzenkamp et al. [52]. The signs of the couplings are related to the value of the $\pi N N$ coupling, for which we use $g_{\pi N N} / \sqrt{4 \pi}=-3.795$.

The remaining parameters of the model are the form factor cutoffs, $\Lambda$, which could in principle be constrained for the various meson-baryon vertices by data from exclusive or inclusive charmed baryon production. In practice, such data are rather limited, and the most direct constraints come from inclusive $\Lambda_{c}$ production in proton-proton scattering, $p p \rightarrow \Lambda_{c} X$, measured by the R680 Collaboration at the ISR [41]. Since it is currently not possible to constrain the cutoffs for the individual charmed meson-baryon configurations, we assume a universal exponential form factor cutoff as in Eq. (18) for all the fluctuations 
listed in Table II, and tune $\Lambda$ to best reproduce the shape and normalization of the inclusive $\Lambda_{c}$ production cross section data. This will place an upper bound on $\Lambda$ and the magnitude of the charmed meson-baryon contribution in the MBM.

Within the same one-boson exchange framework as adopted in the $D N$ and $\bar{D} N$ scattering analyses [38-40], the contribution from charmed meson exchange to the differential cross section for inclusive baryon production in $p p$ scattering can be written [45]

$$
E \frac{d^{3} \sigma}{d^{3} \boldsymbol{p}}=\frac{\bar{y}}{\pi} \frac{d^{2} \sigma}{d \bar{y} d k_{\perp}^{2}}=\frac{\bar{y}}{\pi} \sum_{M}\left|\phi_{B M}\left(\bar{y}, k_{\perp}^{2}\right)\right|^{2} \sigma_{\text {tot }}^{M p}(s y),
$$

where $E$ is the energy of the proton beam, and the sum over $M$ includes incoherent contributions from processes involving the exchange of meson $M$ leading to a final baryon $B$. The total meson-proton cross section $\sigma_{\text {tot }}^{M p}$ here is evaluated at the meson energy sy, with $s$ being the total $p p$ invariant mass squared. For the case of $\Lambda_{c}^{+}$production, the sum is restricted to the $\bar{D}^{0}$ and $\bar{D}^{* 0}$ mesons. The $k_{\perp}^{2}$-integrated cross section for $\Lambda_{c}^{+}$production is then given by

$$
\frac{d \sigma}{d \bar{y}}=\sum_{M=D, D^{*}} f_{\Lambda_{c}^{+} M}(\bar{y}) \sigma_{\text {tot }}^{M p}(s y) .
$$

Note that in Ref. [53] this cross section is defined with an additional factor $(\pi / \bar{y})$ on the right hand side. For the total charmed meson-proton cross section $\sigma_{\text {tot }}^{M p}$ we take a constant value, as suggested by the analysis of pion-nucleon scattering [45], where $\sigma_{\text {tot }}^{\pi p} \approx \sigma_{\text {tot }}^{\rho p}$. Adopting a similar approach to the strange and charmed meson cross sections, we have

$$
\sigma_{\mathrm{tot}}^{D p} \approx \sigma_{\mathrm{tot}}^{D^{*} p} \approx \sigma_{\mathrm{tot}}^{\bar{K} p} \approx(20 \pm 10) \mathrm{mb}
$$

where the value of the $\bar{K} p$ total cross section is taken from Ref. [54], and we assign a conservative $50 \%$ uncertainty on the central value.

Using Eqs. (25) and (26), the calculated cross section is shown in Fig. 2(a) at the kinematics of the $\Lambda_{c}^{+}$production data from the R608 Collaboration [41] at the ISR. The kinematical coverage of the ISR data was restricted to $k_{\perp} \leq 1.1 \mathrm{GeV}$, which we impose in the computed cross section. For the central value of the $D p$ total cross section, $\sigma_{\text {tot }}^{D p}=20 \mathrm{mb}$, the best fit value of the cutoff parameter is found to be $\Lambda=(2.89 \pm 0.04) \mathrm{GeV}$, which gives a good fit to both the overall normalization and the shape of the inclusive $\Lambda_{c}^{+}$data. Including the uncertainty in the $D p$ cross section from Eq. (26), the cutoff becomes $\Lambda=(3.0 \pm 0.2) \mathrm{GeV}$.

Note that in calculating the inclusive $\Lambda_{c}^{+}$production cross section we have included the possibility that higher-mass baryons such as $\Lambda_{c}^{*}$ and $\Sigma_{c}^{*}$ are produced and subsequently decay 

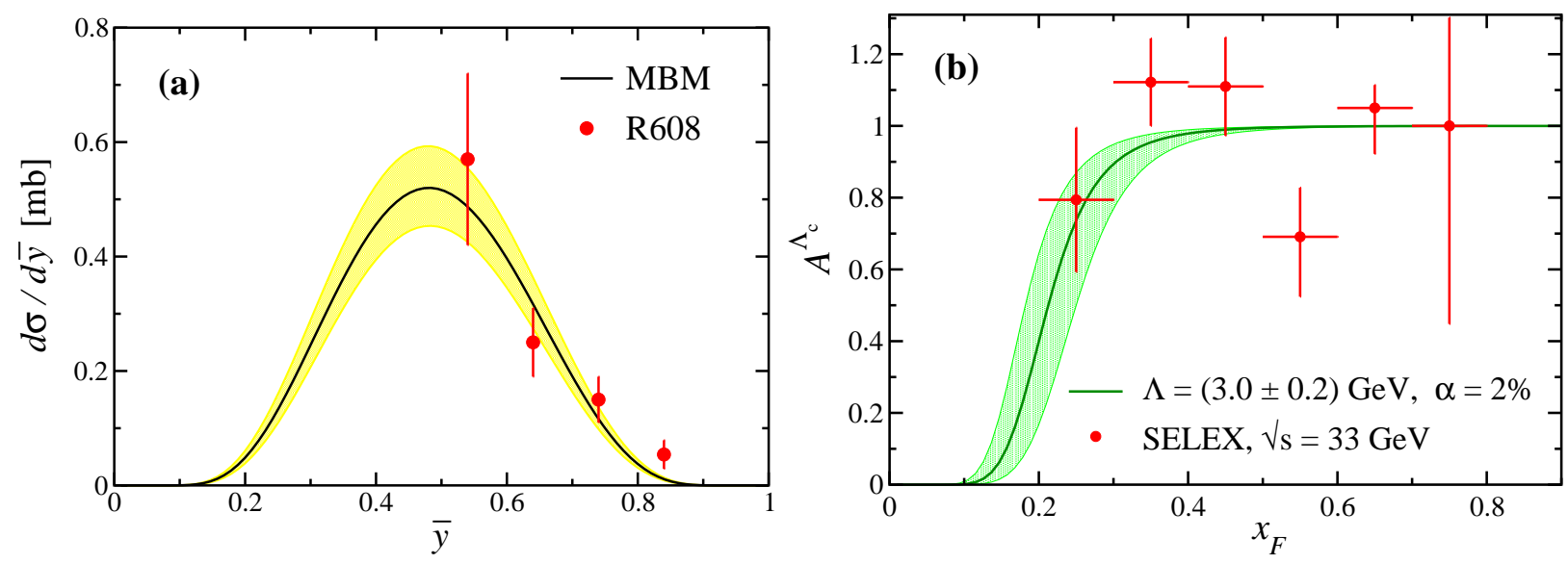

FIG. 2: (color online) (a) Differential cross section $d \sigma / d \bar{y}$ for the inclusive charm production reaction $p p \rightarrow \Lambda_{c}^{+} X$ as a function of the momentum fraction $\bar{y}$ carried by the $\Lambda_{c}^{+}$. The MBM cross section (solid) is computed using the central value for the $D p$ cross section $\sigma_{\text {tot }}^{D p}=20 \mathrm{mb}$, and the resulting error band (shaded) represents the purely statistical uncertainty. The data (red circles) are from the R608 collaboration at the ISR [41]. (b) Charge asymmetry $A^{\Lambda_{c}}$ for $\Lambda_{c}^{+} / \bar{\Lambda}_{c}^{-}$production in the MBM (solid), using the $x_{F}$ dependence of the $\bar{\Lambda}_{c}$ cross section in Eq. (29), compared with data from the SELEX Collaboration [42].

to a $\Lambda_{c}^{+}$, using the relevant branching ratios for the decays. With the coupling constants from Table II, we find that the dominant contribution to inclusive $\Lambda_{c}$ production arises from the state $\bar{D}^{* 0} \Lambda_{c}^{+}$. This is in contrast to earlier analyses, where the largest contribution was assumed to be from the lowest-energy $\bar{D}^{0} \Lambda_{c}^{+}$state. As we discuss below, this will have significant ramifications for intrinsic charm production in electromagnetic reactions.

Note also that the original data from Ref. [41] were recorded in terms of the variable $x_{F}=2 p_{\Lambda}^{0} / \sqrt{s}$, which in general differs from the momentum fraction $\bar{y}$ that scales the calculations of the MBM. It can be shown, however, that at high energies (i.e., $s \gg M_{B}^{2}, k_{\perp}^{2}$ ), one has $\bar{y} \rightarrow x_{F}$.

More recently the SELEX Collaboration at Fermilab [42] has produced data on the charge asymmetry for inclusive $\Lambda_{c}^{+}$and $\bar{\Lambda}_{c}^{-}$production in the scattering of $540 \mathrm{GeV}$ protons from copper and carbon targets,

$$
A^{\Lambda_{c}}\left(x_{F}\right)=\frac{\sigma^{\Lambda_{c}}\left(x_{F}\right)-\sigma^{\bar{\Lambda}_{c}}\left(x_{F}\right)}{\sigma^{\Lambda_{c}}\left(x_{F}\right)+\sigma^{\bar{\Lambda}_{c}}\left(x_{F}\right)},
$$

where $\sigma^{\Lambda_{c}}\left(x_{F}\right) \equiv d \sigma^{\Lambda_{c}} / d x_{F}$. While the contribution to the production of $\Lambda_{c}^{+}$can be calcu- 
lated in the MBM, the computation of the asymmetry $A^{\Lambda_{c}}$ requires in addition an estimate of the $\bar{\Lambda}_{c}$ cross section. Following Ref. [42] we approximate this using a simple monomial parametrization. Furthermore, we assume that the $\Lambda_{c}^{+}$cross sections can be written as the sum of valence and sea components, with the generation of the former described by the nonperturbative MBM and dominating at intermediate and high values of $x_{F}$, and the latter concentrated at small $x_{F}$,

$$
\frac{d \sigma^{\Lambda_{c}}}{d x_{F}}=\frac{d \sigma_{(\mathrm{val})}^{\Lambda_{c}}}{d x_{F}}+\frac{d \sigma_{(\mathrm{sea})}^{\Lambda_{c}}}{d x_{F}}
$$

where

$$
\begin{aligned}
& \frac{d \sigma_{(\mathrm{val})}^{\Lambda_{c}}}{d x_{F}} \approx \sigma_{0} \sum_{M} f_{\Lambda_{c} M}\left(x_{F}\right) \\
& \frac{d \sigma_{(\mathrm{sea})}^{\Lambda_{c}}}{d x_{F}} \equiv \frac{d \sigma^{\bar{\Lambda}_{c}}}{d x_{F}} \approx \bar{\sigma}_{0}\left(1-x_{F}\right)^{\bar{n}}
\end{aligned}
$$

In Eq. (29a) the factor $\sigma_{0}$ corresponds to the total meson-proton cross section in Eq. (25), which we take to be independent of the flavor and spin of the meson, as in Eq. (26), while $\bar{\sigma}_{0}$ is a normalization parameter for the corresponding $\bar{\Lambda}_{c}$ production cross section. Using Eqs. (29), the asymmetry in Eq. (27) can then be written

$$
A_{\Lambda_{c}}\left(x_{F}\right)=\frac{\sum_{M} f_{\Lambda_{c} M}\left(x_{F}\right)}{\sum_{M} f_{\Lambda_{c} M}\left(x_{F}\right)+2 \alpha\left(1-x_{F}\right)^{\bar{n}}},
$$

where $\alpha=\bar{\sigma}_{0} / \sigma_{0}$ is the ratio of the sea to valence contributions to the $\Lambda_{c}$ cross sections. For $\bar{\Lambda}_{c}$ production induced by $\Sigma^{-}$beams, the SELEX Collaboration found for the exponent $\bar{n} \approx 6.8$, which we assume also for the $x_{F}$ dependence of the proton induced cross section in Eq. (29b). Using the MBM cutoff parameter $\Lambda=(3.0 \pm 0.2) \mathrm{GeV}$, a good fit to the SELEX charge asymmetry data can then be obtained with $\alpha \approx 2.0 \%$, as illustrated in Fig. 2(b). We should note, however, that $\Lambda_{c}$ charge asymmetry data are rather sensitive to the form of the $\bar{\Lambda}_{c}$ cross section, so that agreement with the SELEX data should not be considered as a stringent test of the MBM; rather, with an appropriate choice of parameter $\alpha$ the model is able to accommodate the empirical results.

Having constrained the scale parameter for the meson-baryon form factor by the inclusive $\Lambda_{c}$ production data, and with the coupling constants for the various meson-baryon states given in Table II, we are now able to compute the meson-baryon splitting functions in Eq. (10), which we consider in the following section. 


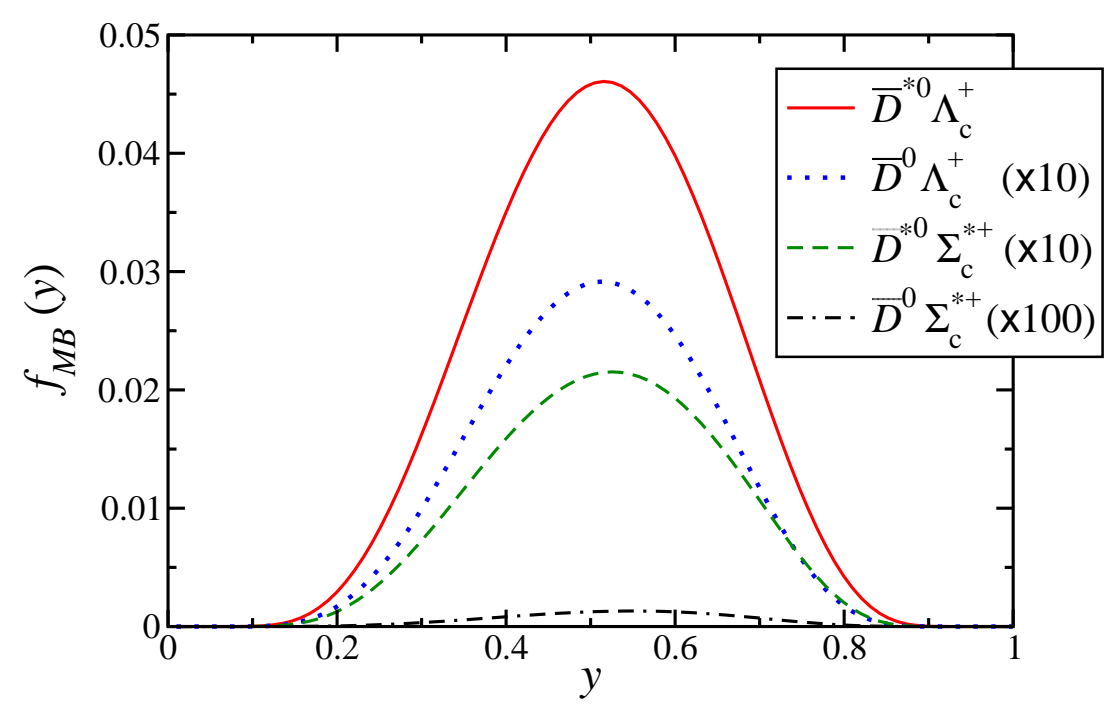

FIG. 3: (color online) Splitting functions for the four basic dissociations of a proton into charmed meson-baryon states, for the spin- 1 meson + spin- $1 / 2$ baryon state $\bar{D}^{* 0} \Lambda_{c}^{+}$(red solid), spin-0 meson + spin- $1 / 2$ baryon state $\bar{D}^{0} \Lambda_{c}^{+}$(scaled $\times 10$, blue dotted), spin- 1 meson + spin- $3 / 2$ baryon state $\bar{D}^{* 0} \Sigma_{c}^{*+}\left(\right.$ scaled $\times 10$, green dashed), and spin- 0 meson + spin-3/2 baryon state $\bar{D}^{0} \Sigma_{c}^{*+}$ (scaled $\times 100$, black dot-dashed). A universal exponential cutoff mass $\Lambda=3 \mathrm{GeV}$ is used with the couplings from Table II.

\section{B. Phenomenology of charmed meson-baryon splitting functions}

The complete set of the four basic splitting functions representing the dissociation of a proton to charmed meson-baryon states $p \rightarrow D B$ (pseudoscalar meson + octet baryon), $D^{*} B$ (vector meson + octet baryon), $D B^{*}$ (pseudoscalar meson + decuplet baryon) and $D^{*} B^{*}$ (vector meson + decuplet baryon) is illustrated in Fig. 3. The functions are shown for the neutral $\bar{D}^{0}$ and $\bar{D}^{* 0}$ mesons, and all the other charge states in Table II can be obtained using appropriate Clebsch-Gordan coefficients. For the best fit value of the universal cutoff parameter $\Lambda=3 \mathrm{GeV}$ from the inclusive $\Lambda_{c}^{+}$production data, the $\bar{D}^{* 0} \Lambda_{c}^{+}$contribution is found to be dominant, an order of magnitude larger than the corresponding $\bar{D}^{0} \Lambda_{c}^{+}$and $\bar{D}^{* 0} \Sigma_{c}^{*+}$ contributions. The $\bar{D}^{0} \Sigma_{c}^{*+}$ contribution is two orders of magnitude smaller still, and effectively plays no role in the phenomenology. To a good approximation, therefore, one can represent the total charm distribution in the proton by the single $\bar{D}^{* 0} \Lambda_{c}^{+}$state.

Interestingly, the shapes of the various charmed meson-baryon distributions $f_{M B}(y)$ are rather similar, peaking just above $y=1 / 2$. This is in contrast to the distributions in the light 
flavor sector, where the corresponding $\pi N$ splitting function is considerably more skewed in $y[44,45]$. The skewedness arises from the large difference in mass between the pion and nucleon in the dissociation, whereas the masses of both the charmed meson and baryon are of the order $\sim 2 \mathrm{GeV}$. This is also one reason why the lowest mass $\pi N$ configuration is the dominant one in the pion sector, unlike the lowest mass charmed state $\bar{D}^{0} \Lambda_{c}^{+}$, which as Fig. 3 indicates gives a significantly smaller contribution than the $\bar{D}^{* 0} \Lambda_{c}^{+}$. The dominance of the $\mathrm{SU}(2)$ flavor sector by the $\pi N$ state is ensured by the relatively large energy gap between higher mass configurations involving $\rho$ mesons or $\Delta$ baryons, whereas no significant energy gap exists between the various states in the charm sector.

To explore further the origin of the dominance of the $\bar{D}^{* 0} \Lambda_{c}^{+}$contribution, we note the relatively strong coupling to the vector meson state, particularly for the tensor coupling term, as seen in Table II, where the tensor to vector coupling ratio is $f_{D^{*} \Lambda_{c} N} / g_{D^{*} \Lambda_{c} N}=3.26$ [38-40]. This is analogous to the large tensor coupling for the $\rho$ meson in one-boson exchange models of the $N N$ interaction [50], where in the Bonn-Jülich model, for instance, one has an even larger tensor/vector ratio, $f_{\rho N N} / g_{\rho N N}=6.1$ [55]. The individual vector, tensor, and vector-tensor interference contributions to the $f_{\bar{D}^{* 0} \Lambda_{c}^{+}}$splitting function are shown in Fig. 4, for the universal $\Lambda=3 \mathrm{GeV}$ exponential cutoff as in Fig. 3. Using the charm couplings from Table II, the tensor contribution clearly dominates over the vector term. This feature is preserved even if one uses the $\mathrm{SU}(2)$ couplings from the $\rho$ exchange in the $N N$ analysis instead of the SU(4) couplings [38-40] (but with the same charm hadron masses). In particular, since the $\mathrm{SU}(4)$ vector coupling $g_{D^{*} \Lambda_{c} N}^{2} / 4 \pi=2.53$ is around 5 times larger than that found for the $\rho$ from $N N$ analyses, $g_{\rho N N}^{2} / 4 \pi=0.55$ [55], the vector contribution to the charm splitting function is significantly larger than for the $\mathrm{SU}(2)$ coupling case. This is compensated somewhat by the $\sim 2$ times smaller $\mathrm{SU}(4)$ tensor/vector ratio, making the total contribution to the charmed vector meson splitting function $f_{\bar{D}^{* 0} \Lambda_{c}^{+}}(y)$ similar.

At the effective Lagrangian level, the large tensor contribution is associated with the additional momentum dependence induced by the derivative coupling in the tensor interaction, which is a general feature of couplings to states with higher spin [see Eq. (A7)]. This additional momentum dependence can have a significant impact on the relative importance of various charmed meson-baryon transitions, as is evident from the form of the splitting function in Eq. (19). The effect of the momentum dependence of the meson-baryon vertices on the splitting functions can be illustrated even more dramatically by considering the 


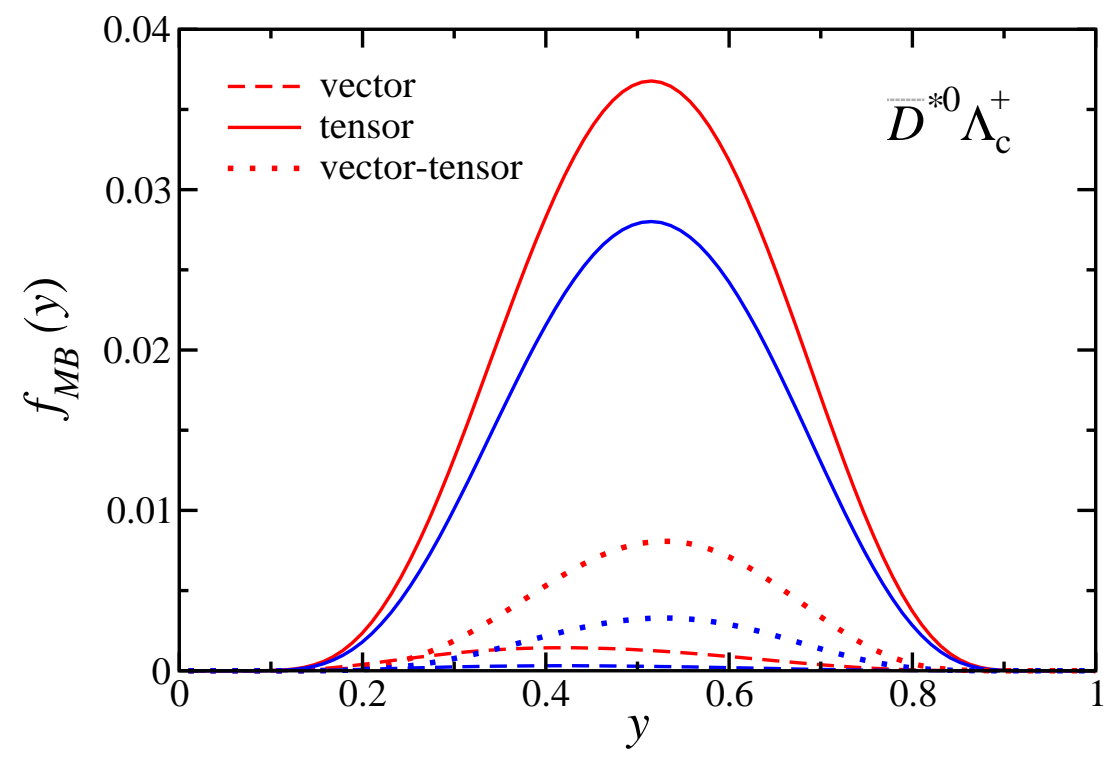

FIG. 4: (color online) Contributions to the $p \rightarrow \bar{D}^{* 0} \Lambda_{c}^{+}$splitting function from the vector (dashed), tensor (solid) and vector-tensor interference (dotted) terms in Eq. (19). The curves are computed for a cutoff mass $\Lambda=3 \mathrm{GeV}$ with charm coupling constants from SU(4) symmetry (red) and using the corresponding $\mathrm{SU}(2)$ values for $\rho N N$ (blue).

normalizations $\langle n\rangle_{M B}=\int d y f_{M B}(y)$ as a function of the cutoff $\Lambda$. These are displayed in Fig. 5(a) for the four charmed states shown in Fig. 3, together with the sum over all contributions. At the best fit value of $\Lambda \sim 3 \mathrm{GeV}$, the lowest mass vector state $\bar{D}^{* 0} \Lambda_{c}^{+}$ makes up around $70 \%$ of the total charm normalization of $\langle n\rangle_{M B}^{(\text {charm })}=2.40 \%$. Including the uncertainty on the cutoff (indicated by the shaded band), the total charm normalization ranges from $\approx 1.04 \%$ to $\approx 4.87 \%$.

While the variation of the charm splitting functions with the choice of $\mathrm{SU}(4)$ or $\mathrm{SU}(2)$ couplings was found in Fig. 4 to be relatively mild, a significant effect is seen for the dependence of the splitting functions on the hadron masses. In Fig. 5(b) the normalizations $\langle n\rangle_{M B}$ of splitting functions to pseudoscalar and vector mesons are illustrated for the light-quark, strange and charmed sectors, using the $\mathrm{SU}(2)$ coupling constants for $\pi N N$ and $\rho N N$ listed above. The curves labeled " $K \Lambda$ " and " $K^{*} \Lambda$ " are obtained from the $\pi N$ and $\rho N$ splitting functions by replacing the pion and recoil baryon masses with the appropriate kaon and hyperon masses, and those labeled " $D \Lambda_{c}$ " and those labeled " $D^{*} \Lambda_{c}$ " are obtained by using the corresponding charmed meson and baryon masses. For small values of the cutoff, the normalizations of the pseudoscalar meson-baryon states is larger than for the vector meson 

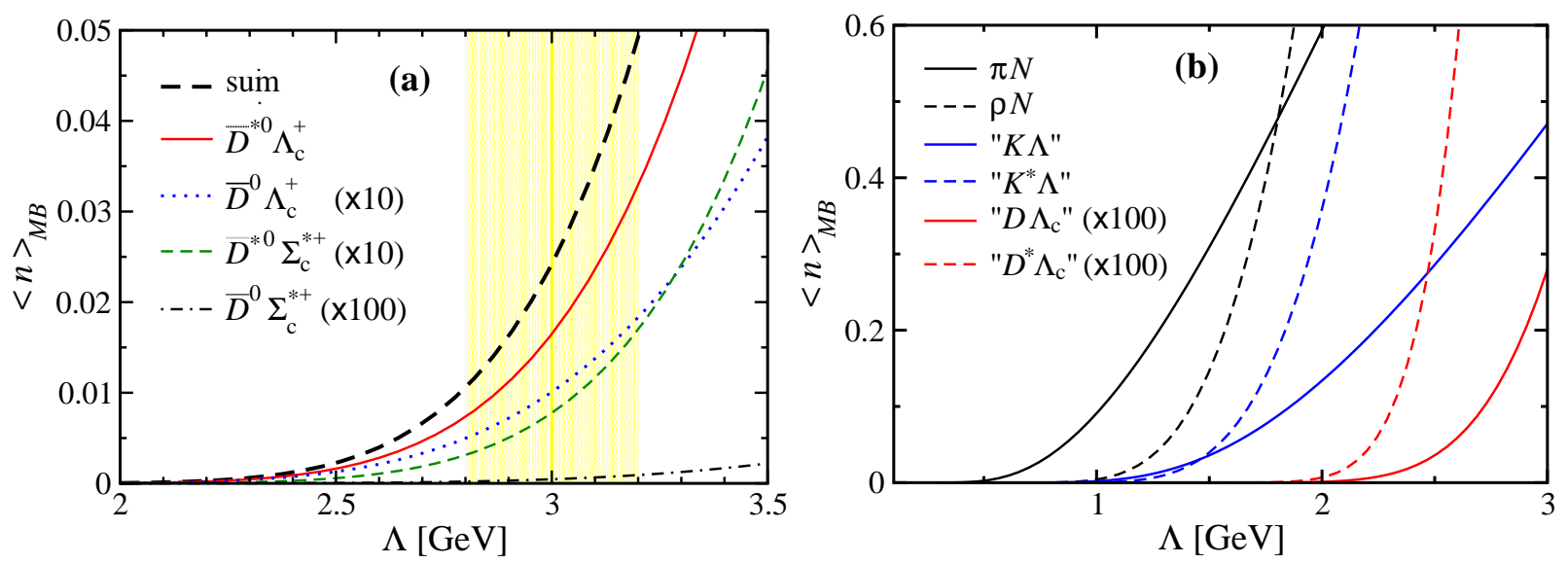

FIG. 5: (color online) (a) Normalizations $\langle n\rangle_{M B}$ of the charmed meson-baryon splitting functions as a function of the form factor cutoff $\Lambda$, for the states $\bar{D}^{* 0} \Lambda_{c}^{+}$(red solid), $\bar{D}^{0} \Lambda_{c}^{+}$(scaled $\times 10$, blue dotted $), \bar{D}^{* 0} \Sigma_{c}^{*+}($ scaled $\times 10$, green dashed $)$, and $\bar{D}^{0} \Sigma_{c}^{*+}$ (scaled $\times 100$, black dot-dashed , as well as the sum of all contributions (black dashed). The (yellow) shaded band represents the uncertainty on the cutoff obtained from fits to inclusive $\Lambda_{c}^{+}$production data. (b) Normalizations of the splitting functions to pseudoscalar (solid) and vector (dashed) mesons computed with $\mathrm{SU}(2)$ sector $(\pi N$ and $\rho N)$ masses (black), $\mathrm{SU}(3)$ masses, denoted by " $K \Lambda$ " and " $K^{*} \Lambda$ " (blue), and $\mathrm{SU}(4)$ masses, denoted by " $D \Lambda_{c}$ " and " $D^{*} \Lambda_{c}$ " (scaled $\times 100$, red), all for the same $\mathrm{SU}(2)$ couplings.

states, but with increasing $\Lambda$ the contributions from the vector meson states eventually dominate. With increasing hadron masses the cross-over point between the pseudoscalar and vector meson states occurs at progressively smaller $\Lambda$ values. Neglecting differences between the coupling constants (which are small if quark model symmetries are assumed), the size of hadronic masses relative to the cutoff scale $\Lambda$ is the main determinant of the balance between the pseudoscalar and vector states for a given flavor sector.

The best fit value $\Lambda=3 \mathrm{GeV}$ for the charmed splitting functions corresponds to a region where the vector meson term clearly dominates over the pseudoscalar meson contribution. Had we found a significantly softer cutoff $\Lambda \sim 1 \mathrm{GeV}$, the pseudoscalar contribution would have dominated, although for such values the total charm contribution would be negligible. Since only a single charmed baryon production cross section was available to constrain the charm splitting functions, only a single parameter $\Lambda$ could be determined. The existence of data for various charmed channels, on the other hand, would allow the cutoffs to be determined for individual meson-baryon states. This could in principle lead to hard form 
factor cutoffs for some states and soft cutoffs for others, which would affect the degree to which the charmed vector meson states dominate the splitting functions. The results of our MBM calculations imply that the production of charmed mesons in $p p$ reactions would occur almost entirely through $D^{*}$ mesons, with subsequent decays of $D^{*}$ to $D$ mesons.

\section{CHARM DISTRIBUTIONS IN CHARMED HADRONS}

Within the two-step, meson-baryon convolution picture in Eqs. (8), the charm and anticharm distributions in the nucleon require knowledge of the $c$ and $\bar{c}$ distributions in the charmed baryon and meson. In applications of the MBM to the light quark sector, where the $\bar{u}$ and $\bar{d}$ distributions arise from the pionic component of the nucleon wave function, one can use data from measurements of the pion structure function $[56,57]$. There is even some empirical information on the kaon structure function from the Drell-Yan reaction [58], which has been utilized in calculations of strange quark distributions in the nucleon [59, 60]. In contrast, nothing at all is known about the partonic structure of charmed hadrons, so that in practice these need to be modelled.

In the literature estimates of the distributions of heavy quarks in heavy hadrons have been made using the heavy quark limit [15], and within a scalar constituent quark model [21]. Here we combine several features of these approaches in constructing a relativistic quark model, with the correct spin degrees of freedom, that parallels the splitting function calculations of Sec. IV. We apply the time-ordered perturbation theory framework in the IMF at the parton level, defining the IMF momentum fraction $\hat{y}=\hat{k}_{L} / P_{L}$ to be the ratio of the longitudinal momentum of the constituent quark or antiquark $\left(\hat{k}_{L}\right)$ to that of the parent charmed meson or baryon $\left(P_{L}\right)$. Convolution with the leading twist point-like structure of constituent quarks gives distributions as functions of the quark-level Bjorken limit variable, denoted here by $z$ to prevent confusion with quark distributions in the proton. In the following we summarize the results for the $\bar{c}$ distributions in $D$ and $D^{*}$ mesons, and the $c$ distributions in the $\Lambda_{c}$ and $\Sigma_{c}^{*}$ baryons; technical details of the calculations are presented in Appendix B. 


\section{A. Anticharm in charmed mesons}

The distribution of a relativistic $\bar{c}$ quark in a pseudoscalar $D$ meson, with a spectator $u$ or $d$ quark, can be computed in analogy with the $p \rightarrow D \Lambda_{c}$ splitting function in Eq. (16). Using a pseudoscalar meson-quark-antiquark vertex parametrized by the structure $\gamma_{5} G(\hat{s})$, where $G(\hat{s})$ is the $D-\bar{c}-q$ vertex function $(q=u, d)$, the distribution of anticharm quarks in the $D$ meson is given by

$$
\bar{c}_{D}(z)=\frac{N_{D}}{16 \pi^{2}} \int_{0}^{\infty} \frac{d \hat{k}_{\perp}^{2}}{[z(1-z)]^{2}} \frac{|G(\hat{s})|^{2}}{\left(\hat{s}-m_{D}^{2}\right)^{2}}\left[\hat{k}_{\perp}^{2}+\left(z m_{q}+(1-z) m_{\bar{c}}\right)^{2}\right],
$$

where the integration is over the transverse momentum $\hat{k}_{\perp}^{2}$ of the interacting heavy quark, and $z$ is the Bjorken scaling variable of the heavy quark inside the charmed hadron. The total invariant mass squared of the $\bar{c} q$ pair is defined [see also Eq. (7)] for the corresponding invariant mass of the meson-baryon system) as

$$
\hat{s}\left(z, \hat{k}_{\perp}^{2}\right)=\frac{m_{\bar{c}}^{2}+\hat{k}_{\perp}^{2}}{z}+\frac{m_{q}^{2}+\hat{k}_{\perp}^{2}}{1-z}
$$

where $m_{\bar{c}}$ is the constituent anticharm quark mass, $m_{q}$ is the mass of the (light) spectator quark, and $N_{D}$ is the overall normalization factor determined by the valence normalization condition,

$$
\int_{0}^{1} d z \bar{c}_{D}(z)=1
$$

For point particles, the ultraviolet behavior of the $\hat{k}_{\perp}^{2}$ integration would be logarithmically divergent for the $\bar{c}_{D}(z)$ distribution in Eq. (31). The divergence can be regulated by defining the vertex function $G(\hat{s})$ to suppress contributions from large parton momenta. Following Sec. IV, we can use, for example, an exponential functional dependence on $\hat{s}$,

$$
G(\hat{s})=\exp \left[-\left(\hat{s}-m_{D}^{2}\right) / \hat{\Lambda}^{2}\right]
$$

with $\hat{\Lambda}$ serving the role of a corresponding momentum cutoff on the partonic quark-antiquark system.

At low momenta, on the other hand, a mass singularity can arise in the energy denominator $\left(\hat{s}-m_{D}^{2}\right)^{-2}$ in the infrared limit $\left(\hat{k}_{\perp}^{2} \rightarrow 0\right)$ for physical quark masses $m_{q}$ and $m_{\bar{c}}$. A simple solution adopted by Pumplin [21] was to assume an artificially large effective mass for the anticharm quark, $m_{\bar{c}}^{\text {eff }}$, and a large constituent quark mass for the spectator $u$ or $d$ 
quark, $m_{q}^{\text {eff }}$, such that

$$
m_{\bar{c}}^{\mathrm{eff}}+m_{q}^{\mathrm{eff}}>m_{D}
$$

In our numerical analysis we fix the effective charm mass to be $m_{\bar{c}}^{\text {eff }}=1.75 \mathrm{GeV}$ and the light constituent quark mass $m_{q}^{\mathrm{eff}}=M / 3=0.31 \mathrm{GeV}$, similar to that used in Ref. [21], which is sufficient to remove the propagator singularity.

An alternative method to avoid the pole is to utilize a form factor that simulates confinement by directly cancelling the singular denominator, similar to that advocated in Ref. [48]. A form that satisfies this is

$$
G(\hat{s})=\left(\hat{s}-m_{D}^{2}\right) \exp \left[-\left(\hat{s}-m_{D}^{2}\right) / \hat{\Lambda}^{2}\right]
$$

An attractive feature of this form of the vertex function is that it permits any values of the quark masses to be used, allowing the partons to be confined without the need for ad hoc constraints to avoid singularities through judicious choice of effective quark masses.

For the $\bar{c}$ distribution in a vector $D^{*}$ meson, there exist in principle both the Dirac and Pauli couplings of the $D^{*}$ to a quark and antiquark, as for the $D^{*} B$ splitting function in Eq. (19). To reduce the number of free parameters in the calculation, we make the simplifying assumption that the $D^{*}$-quark-antiquark coupling is governed by a purely vector interaction, $\gamma_{\alpha} G(\hat{s})$. In this case the distribution of a $\bar{c}$ quark in the $D^{*}$ meson is given by

$$
\begin{array}{r}
\bar{c}_{D^{*}}(z)=\frac{N_{D^{*}}}{16 \pi^{2}} \int_{0}^{\infty} \frac{d \hat{k}_{\perp}^{2}}{[z(1-z)]^{2}} \frac{|G(\hat{s})|^{2}}{\left(\hat{s}-m_{D^{*}}^{2}\right)^{2}}\left[\left(\frac{\hat{k}_{\perp}^{2}+m_{q}^{2}}{m_{D^{*}}^{2}}+(1-z)^{2}\right)\left(\hat{k}_{\perp}^{2}+m_{\bar{c}}^{2}+z^{2} m_{D^{*}}^{2}\right)\right. \\
\left.+\hat{k}_{\perp}^{2}+\left(z m_{q}+(1-z) m_{\bar{c}}\right)^{2}+4 z(1-z) m_{q} m_{\bar{c}}\right], \quad(37)
\end{array}
$$

where the normalization factor $N_{D^{*}}$ is again determined by the valence quark number conservation condition in Eq. (33). As for the $\bar{c}$ distribution in the $D$ meson, for point interactions the integral in Eq. (37) would be divergent, in this case linearly in $\hat{k}_{\perp}^{2}$. Vertex form factors $G(\hat{s})$ such as in Eqs. (34) or (36) would again act to regularize this divergence.

The results for the $\bar{c}$ distributions in the $D$ and $D^{*}$ mesons are illustrated in Fig. 6 for both types of vertex functions $G(\hat{s})$. In the absence of empirical constraints on PDFs in charmed mesons, the partonic cutoff $\hat{\Lambda}$ is a free parameter. However, since for heavy quarks the typical masses of the intermediate states $(D B$ or $\bar{c} q)$ are comparable, to a first approximation we can fix $\hat{\Lambda}$ to the meson-baryon cutoff, $\hat{\Lambda}=\Lambda$. In the effective mass model, 

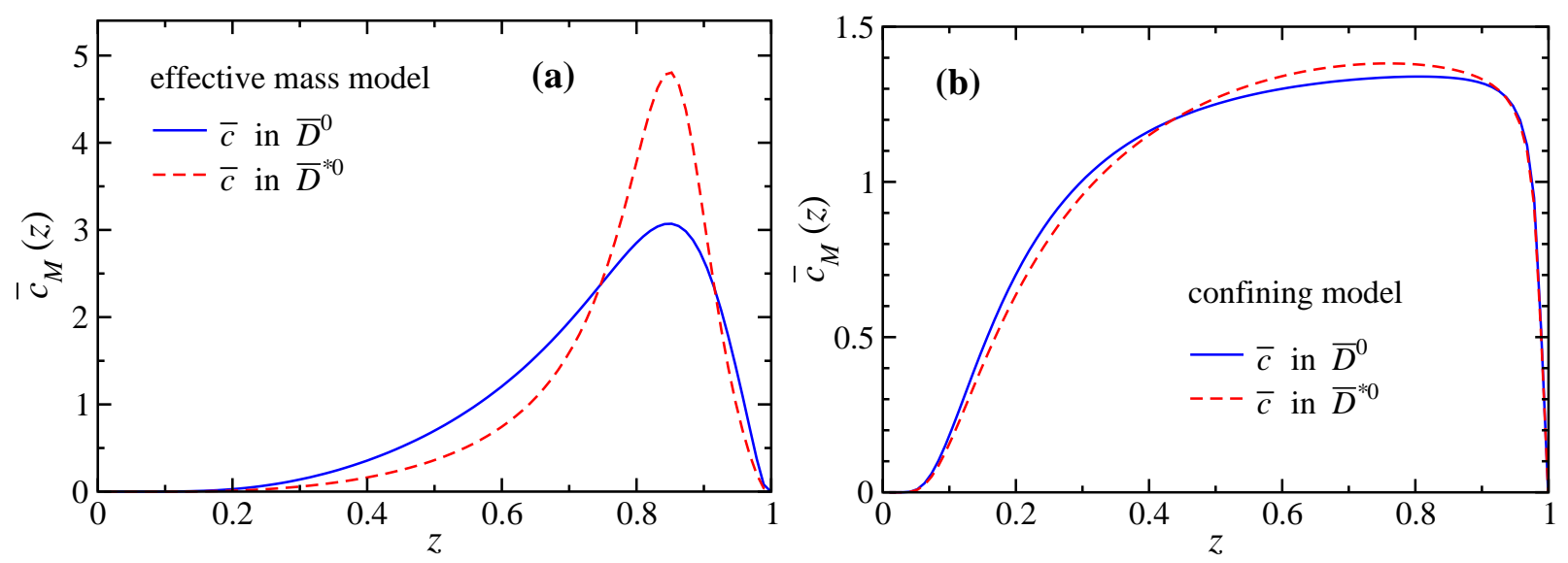

FIG. 6: (color online) Anticharm quark distributions in charmed $D$ (solid) and $D^{*}$ (dashed) mesons, within the (a) effective mass model, with the vertex form factor in Eqs. (34) and (35), and (b) confining model, with the form factor in Eq. (36).

Eqs. (34) and (35), the peak of the anticharm distribution in $z$ reflects the fraction of the meson mass carried by the $\bar{c}$ quark. For both the $D$ and $D^{*}$ mesons, the $\bar{c}$ distribution peaks at $z \sim 0.85$, with the latter being slightly narrower. The distributions in the confining model, Eq. (36), also peak at similarly large momentum fractions, but are significantly broader.

A numerical feature of the effective mass model is the presence of the energy denominator $\propto\left(\hat{s}-m_{D}^{2}\right)^{-2}$, which largely determines the qualitative shapes of the $\bar{c}$ distributions. For specific mass choices, $\hat{s}-m_{D}^{2}$ is minimized at a unique value of $z$, resulting in the stronglypeaked shapes observed in the effective mass model. In the confining model, on the other hand, the energy denominator responsible for this $z$ dependence is suppressed directly such that the resulting distribution shapes no longer possess pronounced maxima. In the effective charm model, however, the closer the energy denominator approaches its pole value, the more "singular" the behavior at the distribution maximum; as such, if we fix the charm and spectator masses according to Eq. (35), the energy denominator approaches the zero pole for heavier hadron masses, producing the more sharply peaked distributions seen in Fig. 6(a).

\section{B. Charm in charmed baryons}

The calculation of the charm quark distributions in charmed baryons proceeds in similar fashion to that for the $D$ and $D^{*}$ mesons, but is more involved since the spectator system consists of two (or more) particles. In practice, however, one can simplify the calculation 
by treating the spectator $q q$ system as an effective "diquark" with a fixed mass $m_{q q}$. For spin-1/2 charmed baryons, in general the spectator diquark state can have either spin 0 or spin 1, with corresponding scalar and pseudovector vertex functions describing the momentum dependence. The spin of the spectator diquark can affect the spin and flavor dependence of the associated parton distribution; for example, the suppression of the $d / u$ ratio in the proton at large $x$ is usually attributed to a higher energy of the spin-1 diquark in the proton compared with the spin-0 diquark $[15,61]$. Since here we are concerned with the total effect on the charm quark distribution, rather than the flavor dependence, it will be sufficient to consider only the leading contribution arising from the scalar spectators, for which we take an effective mass of $m_{q q}=1 \mathrm{GeV}$.

The charm quark distribution in a spin-1/2 charmed baryon $B\left(B=\Lambda_{c}\right.$ or $\left.\Sigma_{c}\right)$ with a scalar $q q$ spectator is then given by

$$
c_{B}(z)=\frac{N_{B}}{16 \pi^{2}} \int_{0}^{\infty} \frac{d \hat{k}_{\perp}^{2}}{z^{2}(1-z)} \frac{|G(\hat{s})|^{2}}{\left(\hat{s}-M_{B}^{2}\right)^{2}}\left[\hat{k}_{\perp}^{2}+\left(m_{c}+z M_{B}\right)^{2}\right],
$$

where for the charm quark mass we take $m_{c}=m_{\bar{c}}$, and the invariant mass squared of the quark-diquark system here is defined as

$$
\hat{s}\left(z, \hat{k}_{\perp}^{2}\right)=\frac{m_{c}^{2}+\hat{k}_{\perp}^{2}}{z}+\frac{m_{q q}^{2}+\hat{k}_{\perp}^{2}}{1-z} .
$$

The functional form of the $B-c-q q$ vertex function $G(\hat{s})$ is taken to be the same as for the $D-\bar{c}-q$ function in the models of Eqs. (34) and (36), and the normalization constant $N_{B}$ determined from an analogous valence charm quark number condition to that in Eq. (33),

$$
\int_{0}^{1} d z c_{B}(z)=1
$$

Note that for the $\Sigma_{c}^{++}$baryon, the $u$ spectator diquark has spin 1, so that the calculation of its $c$ quark distribution here is approximated by neglecting the diquark's spin structure. While it is straightforward to include both spin-0 and spin-1 diquark contributions, in analogy with the spin structures discussed in Sec. VA, since the overall contribution from the dissociation of the proton to $D^{-} \Sigma_{c}^{++}$is at least an order of magnitude smaller than for $\bar{D}^{* 0} \Lambda_{c}^{+}$, this will have negligible effect on the numerical results.

For the spin-3/2 $\Sigma_{c}^{*}$ baryons, the charm quark is always accompanied by a spin-1 diquark. Incorporating the fully relativistic Rarita-Schwinger structure for the spin-3/2 state (see 

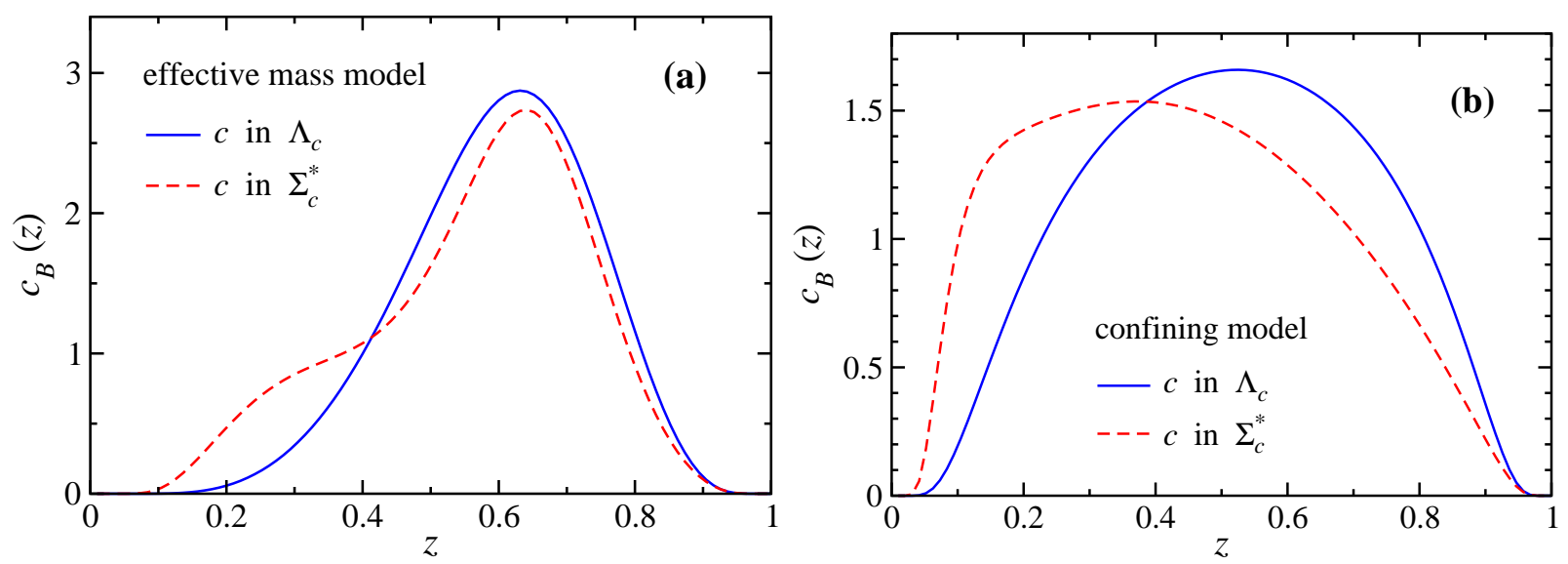

FIG. 7: (color online) Charm distributions in the charmed $\Lambda_{c}$ (solid) and $\Sigma_{c}^{*}$ (dashed) baryons, within (a) the effective mass model, and (b) the confining model.

Appendix B), the charm quark distribution in the $\Sigma_{c}^{*}$ is given by

$$
\begin{aligned}
c_{B^{*}}(z) & =\frac{N_{B^{*}}}{12 \pi^{2} m_{q q}^{2}} \int_{0}^{\infty} \frac{d \hat{k}_{\perp}^{2}}{z(1-z)} \frac{|G(\hat{s})|^{2}}{\left(\hat{s}-M_{B^{*}}^{2}\right)^{2}}\left((\hat{k} \cdot \hat{\Delta})(P \cdot \hat{\Delta})+2 m_{c} M_{B^{*}} \hat{\Delta}^{2}\right. \\
& +\frac{1}{m_{q q}^{2}}\left[m_{c} M_{B^{*}}(\hat{p} \cdot \hat{\Delta})^{2}-(\hat{p} \cdot \hat{\Delta})((\hat{p} \cdot \hat{\Delta})(\hat{k} \cdot \hat{p})-(P \cdot \hat{\Delta})(\hat{k} \cdot \hat{p})-(\hat{k} \cdot \hat{\Delta})(P \cdot \hat{p}))\right. \\
& \left.\left.-(P \cdot \hat{p})(\hat{k} \cdot \hat{p}) \hat{\Delta}^{2}-\frac{P \cdot \hat{k}}{M_{B^{*}}^{2}}\left((P \cdot \hat{p})^{2} \hat{\Delta}^{2}-m_{q q}^{2}(P \cdot \hat{\Delta})^{2}-2(P \cdot \hat{\Delta})(\hat{p} \cdot \hat{\Delta})(P \cdot \hat{p})\right)\right]\right),
\end{aligned}
$$

in which $\hat{p}$ is the momentum of the spectator diquark $q q$, and $\hat{\Delta} \equiv P-\hat{k}$ [see Eq. (A3)].

The resulting $c$ quark distributions in the spin-1/2 and spin-3/2 charmed baryons are illustrated in Fig. 7, using the same numerical values for the masses and cutoffs as in the $\bar{c}$ calculation in the charmed mesons above. Compared with the $\bar{d}$ distributions in $D$ and $D^{*}$, the $c$ quark PDFs are peaked at somewhat smaller values of $z$. In the effective mass model for the $B-c-q q$ vertex function, both the $c$ distributions in $\Lambda_{c}$ and in $\Sigma_{c}^{*}$ are maximal at $z \approx 0.6-0.65$, with a relatively narrow distribution in $z$. The bulge in the $c$ distribution in the $\Sigma_{c}^{*}$ baryon is associated with the more complicated spin algebra compared with the $\Lambda_{c}$. The $c$ distributions with the confining model vertex function are once again somewhat broader, peaking at smaller $z$ values, $z \approx 0.55$ for the $\Lambda_{c}$ baryon and $z \approx 0.4$ for the $\Sigma_{c}^{*}$, with the latter having a sharp drop off at $z \rightarrow 0$. The broader distributions here are generated by the suppression of the energy denominator $\left(\hat{s}-M_{B}^{2}\right)^{-2}$, which is mostly responsible for the strongly-peaked distributions in the effective mass model. In all cases the distributions 
have been normalized to respect the valence quark number sum rule, as in Eq. (40).

Having assembled the various ingredients for the calculation of the convolution expressions in Eqs. (8), in the next section we combine these inputs to compute the $c$ and $\bar{c}$ distributions in the nucleon.

\section{INTRINSIC CHARM IN THE NUCLEON}

Combining the distributions of $c$ and $\bar{c}$ quarks in the charmed mesons and baryons discussed in the previous section with the splitting functions summarized in Sec. IV, here we present the resulting $c$ and $\bar{c}$ distributions in the physical nucleon. We consider in Sec. VIA contributions from the various meson-baryon configurations in the MBM, and the dependence of the results on the models for the charm distributions inside the charm hadrons. We compare our results with other prescriptions for intrinsic charm distributions in Sec. VIB, and with measurements of the charm structure function in Sec. VIC.

\section{A. Intrinsic charm in the MBM}

The contributions to the charm and anticharm quark distributions in the nucleon from various meson-baryon states are presented in Fig. 8, using the confining model for the PDFs in the charmed hadrons (36) with a mass parameter $\Lambda=3 \mathrm{GeV}$. The contributions correspond to the same configurations as in Fig. 3, namely, the dominant $\bar{D}^{* 0} \Lambda_{c}^{+}$state, the $\bar{D}^{0} \Lambda_{c}^{+}$and $\bar{D}^{* 0} \Sigma_{c}^{*+}$ states, as well as the (negligible) $\bar{D}^{0} \Sigma_{c}^{*+}$ contribution. As expected from the magnitudes of the splitting functions in Fig. 3, the $\bar{D}^{*} \Lambda_{c}^{+}$state produces the dominant meson-baryon contribution to charm.

Summing over all the contributions listed in Fig. 1, the total $x c$ and $x \bar{c}$ distributions are shown in Fig. 9, at the input scale $Q^{2}=m_{c}^{2}$ and evolved to $Q^{2}=50 \mathrm{GeV}^{2}$ (which is typical

for charm structure function measurements). Note that the dominant $\bar{D}^{* 0} \Lambda_{c}^{+}$contribution accounts for nearly $70 \%$ of the total. A unique feature of the MBM which is evident in Fig. 9 is the fact that $\bar{c}$ distribution is clearly harder than the $c$ distribution. This is true for every meson-baryon configuration in the MBM, and simply reflects the fact that the charm quark represents a larger fraction of the total mass of the meson than of the baryon. Since the peak in the charm distribution in a hadron is related to the fraction of the hadron mass 

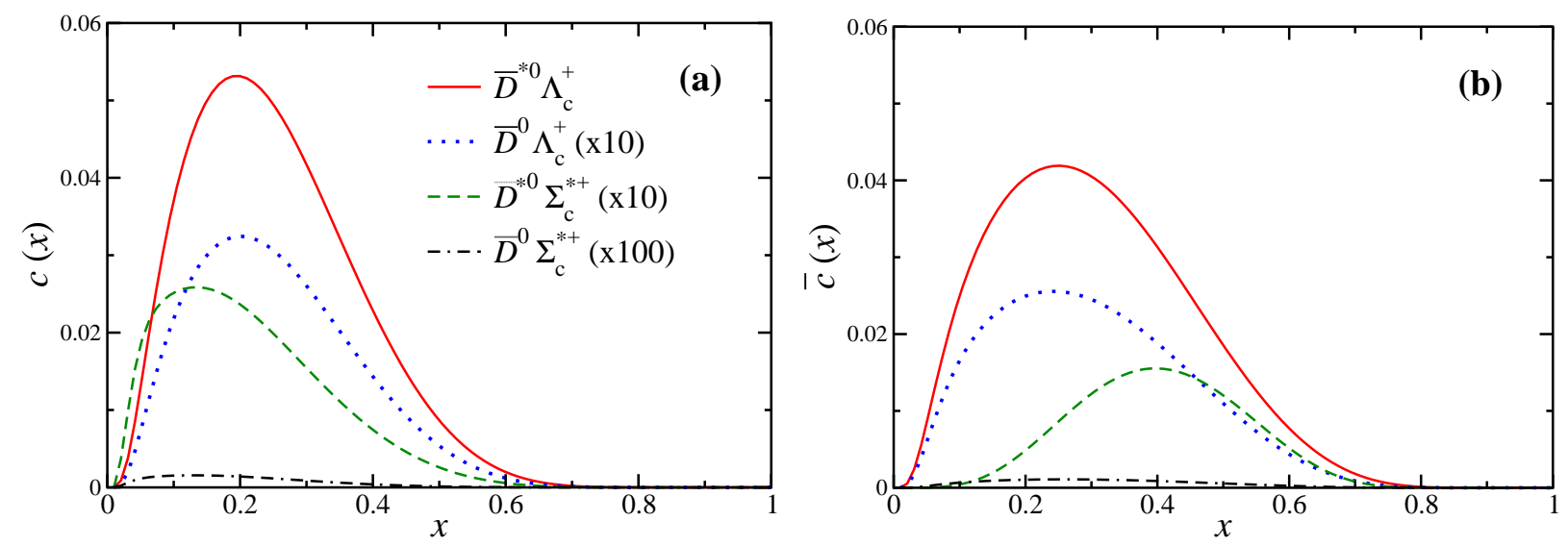

FIG. 8: (color online) (a) Charm and (b) anticharm quark distributions in the nucleon in the MBM, with contributions from the meson-baryon configurations as in Fig. 3: $\bar{D}^{* 0} \Lambda_{c}^{+}$(red solid), $\bar{D}^{0} \Lambda_{c}^{+}$(scaled $\times 10$, blue dotted), $\bar{D}^{* 0} \Sigma_{c}^{*+}$ (scaled $\times 10$, green dashed), and $\bar{D}^{0} \Sigma_{c}^{*+}($ scaled $\times 100$,
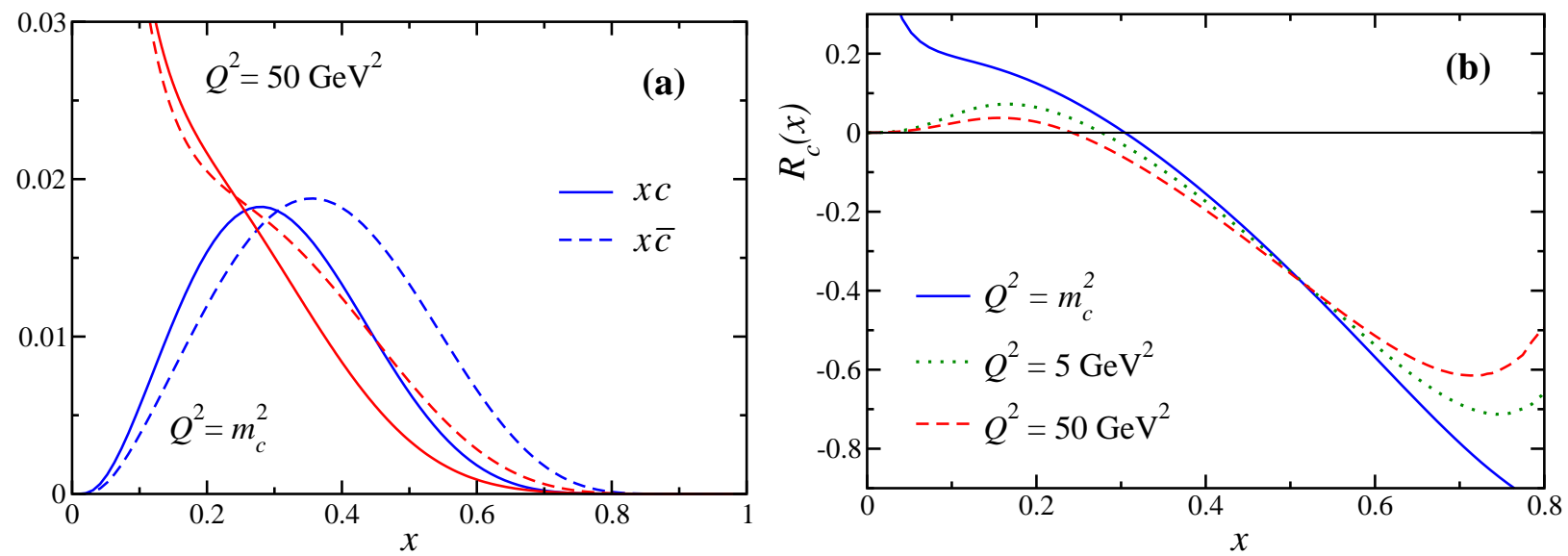

FIG. 9: (color online) (a) Total $x c$ (solid lines) and $x \bar{c}$ (dashed lines) distributions in the MBM with the confining model for the PDFs in the charmed hadrons, Eq. (36), at $Q^{2}=m_{c}^{2}$ (blue) and evolved to $Q^{2}=50 \mathrm{GeV}^{2}$ (red). (b) Corresponding charm-anticharm asymmetry $R_{c}(x)=$ $(c(x)-\bar{c}(x)) /(c(x)+\bar{c}(x))$ at $Q^{2}=m_{c}^{2}$ (solid), $Q^{2}=5 \mathrm{GeV}^{2}$ (dotted), and $Q^{2}=50 \mathrm{GeV}^{2}$ (dashed).

carried by the charm quark, the resulting distribution of $\bar{c}$ in the $\bar{D}$ meson will typically be harder than that for the $c$ in the $\Lambda_{c}$. While it is possible to make the intrinsic $c$ distribution as hard as the $\bar{c}$ distribution in a convolution model, this requires rather unnatural parton distributions inside the baryon and meson states.

To quantify the magnitude of the nonperturbative charm in the nucleon, we can compute 
the total proton momentum carried by charm and anticharm quarks,

$$
P_{c}=C^{(1)}+\bar{C}^{(1)}
$$

where the moments $C^{(1)}$ and $\bar{C}^{(1)}$ are defined in Eqs. (11). For the confining model distributions in the charmed mesons and baryons, the momentum fraction at the input model scale (which is naturally of the order of the charm quark mass) is found to be $P_{c}=1.34_{-0.75}^{+1.35} \%$ for the cutoff mass parameter $\Lambda=(3.0 \pm 0.2) \mathrm{GeV}$ obtained from the inclusive $\Lambda_{c}$ production data, Sec. IV A. Again we note that these first moments differ numerically from the charm multiplicities mentioned in Sec. IV B, where we found $\langle n\rangle_{M B}^{(\text {charm })}=2.40_{-1.36}^{+2.47} \%$. The strong dependence of the total momentum on $\Lambda$ stems from the strong dependence of the dominant meson-baryon splitting function on the hadronic form factor, as seen in Fig. 5(a). In the BHPS model, in contrast, when the charm quark is normalized to $1 \%$ probability in the nucleon, charm quarks carry a momentum fraction $P_{c}=0.57 \%$. For our best fit form factor cutoff mass $\Lambda=3 \mathrm{GeV}$, therefore, nonperturbative charm quarks carry about twice the momentum as in the BHPS model.

Valence quark normalization requires that the first moment of $c-\bar{c}$ vanishes, as in Eq. (15), which follows for any splitting function that obeys the reciprocity relation, Eq. (10). Higher moments, on the other hand, are not required to vanish. In fact, the magnitude of the $c-\bar{c}$ asymmetry can be quantified in terms of the difference of the second moments (momentum carried by charm and anticharm quarks),

$$
\Delta P_{c}=C^{(1)}-\bar{C}^{(1)}
$$

At the model scale, $Q^{2}=m_{c}^{2}$, we find $\Delta P_{c}=-\left(0.13_{-0.08}^{+0.14}\right) \%$ for $\Lambda=(3.0 \pm 0.2) \mathrm{GeV}$. The overall negative values of $\Delta P_{c}$ reflect the fact that in the MBM the $\bar{c}$ distribution is harder than the $c$.

The momentum imbalance of anticharm quarks compared to charm can be estimated from the ratio of the difference $\Delta P_{c}$ to the sum $P_{c}$, for which we find $\Delta P_{c} / P_{c} \approx-10 \%$. As a function of $x$, however, the imbalance is not uniformly distributed. Defining the ratio

$$
R_{c}(x)=\frac{c(x)-\bar{c}(x)}{c(x)+\bar{c}(x)}
$$

from Fig. 9(b) we observe that the relative asymmetry can exceed $50 \%$ at large values of $x$ $(x \gtrsim 0.5)$. The $Q^{2}$ dependence of the ratio indicates relatively mild effects over the large 

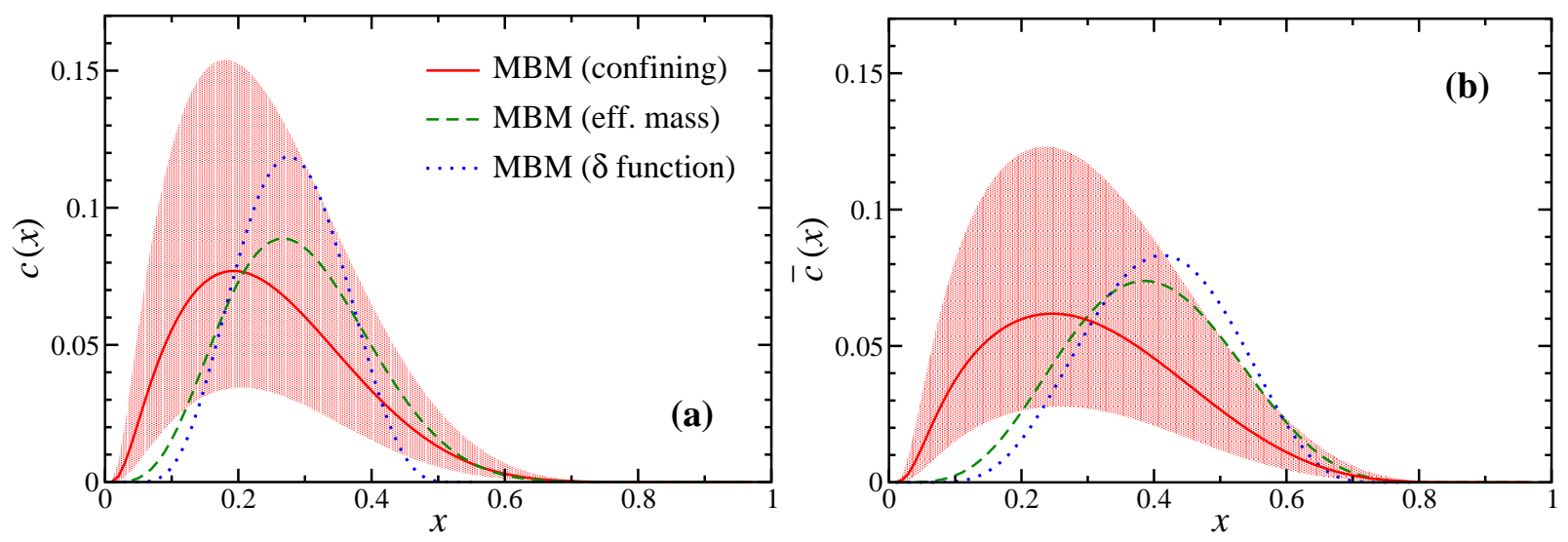

FIG. 10: (color online) Model dependence of the charm distributions in the nucleon for (a) $c(x)$ and $(\mathbf{b}) \bar{c}(x)$, for the MBM with the confining model for the PDFs in charmed hadrons (red solid), the effective mass model (green dashed), and the $\delta$ function model (blue dotted). All distributions use a common value for the cutoff mass of $\Lambda=(3.0 \pm 0.2) \mathrm{GeV}$, with the uncertainty band shown for the confining model.

range considered (up to $Q^{2}=50 \mathrm{GeV}^{2}$ ), with the slope of the asymmetry becoming slightly more shallow with increasing $Q^{2}$. Note that the ratio $R_{c}$ is nonzero at $x=0$ at the model scale, but the effects of perturbative evolution force $R_{c}(x=0)$ to vanish at large $Q^{2}$ values due to the growth of the denominator $c+\bar{c}$.

\section{B. Comparison with other models}

While some of the features of the nonperturbative $c$ and $\bar{c}$ distributions in the MBM are relatively robust, such as the generally harder $x$ dependence compared with the perturbatively generated distributions and the presence of a $c-\bar{c}$ asymmetry, the detailed $x$ dependence does depend on the specifics of the model. To estimate the model dependence of the calculated $c$ and $\bar{c}$ PDFs, we compare the results obtained in the previous section, using the splitting functions from Sec. III and the confining model for the PDFs in the charmed hadrons in Sec. V, with distributions computed under different assumptions and approximations.

Within the same MBM framework, if one uses the effective mass model for the charm PDFs in the charmed mesons and baryons, the resulting $c$ and $\bar{c}$ distributions in the nucleon are slightly harder, especially for the $\bar{c}$, as Fig. 10 illustrates. This generally follows from 
the shape of the $\bar{c}_{M}$ distribution in the confining and effective mass models in Fig. 6, where the latter is more strongly peaked at large values of the parton momentum fraction. The corresponding value of the total nucleon momentum carried by charm and anticharm quarks in the effective mass model is $P_{c}=1.67_{-0.94}^{+1.70} \%$ for cutoff masses $\Lambda=(3.0 \pm 0.2) \mathrm{GeV}$, and $\Delta P_{c}=-\left(0.24_{-0.14}^{+0.28}\right) \%$ for the momentum asymmetry, with the resulting momentum imbalance $\Delta P_{c} / P_{c} \approx-15 \%$. The $c-\bar{c}$ asymmetry in this model is therefore more pronounced than in the confining model.

In a more simplified approach, the $c$ and $\bar{c}$ distributions inside the charmed hadrons were approximated in Ref. [15] by $\delta$ functions centered at the $x$ values corresponding to the fraction of the hadron mass carried by the constituent charm or anticharm quark,

$$
c_{B}(x)=\delta\left(x-x_{B}\right) \text { and } \bar{c}_{M}(x)=\delta\left(x-x_{M}\right) .
$$

From Eqs. (8), the charm and anticharm distributions in the nuclear are then given directly as sums over the various meson-baryon splitting functions,

$$
\begin{aligned}
& c(x)=\sum_{B, M} \frac{1}{x_{B}} f_{B M}\left(\frac{x}{x_{B}}\right), \\
& \bar{c}(x)=\sum_{M, B} \frac{1}{x_{M}} f_{M B}\left(\frac{x}{x_{M}}\right) .
\end{aligned}
$$

Since the masses of the charm quark $\left[m_{c}=\mathcal{O}(1.5 \mathrm{GeV})\right]$ and the $D$ mesons $\left[m_{D}=\right.$ $\mathcal{O}(1.8-2 \mathrm{GeV})]$ are similar, as a first approximation one can take $x_{M} \approx 1$. Similarly, for the fractional mass of the $c$ quark in the charmed baryon, the approximation $x_{B} \approx 2 / 3$ was utilized [15]. In a somewhat more sophisticated approach, one can choose $x_{M}$ and $x_{B}$ to minimize the $\hat{s}$-dependent energy denominator, which depends on the combination $m_{c}^{2} / x+m_{\mathrm{spec}}^{2} /(1-x)$, where the spectator mass $m_{\mathrm{spec}}$ corresponds to the light quark mass $m_{u, d}$ for a meson, and to an effective diquark mass $m_{q q}$ for a baryon. Choosing $m_{c}=1.3 \mathrm{GeV}$, $m_{u, d}=0.313 \mathrm{GeV}$ and $m_{q q}=1 \mathrm{GeV}$, one has

$$
x_{B}=\frac{m_{c}}{m_{c}+m_{q q}} \approx 0.57, \quad x_{M}=\frac{m_{\bar{c}}}{m_{\bar{c}}+m_{u, d}} \approx 0.81 .
$$

For the best fit form factor cutoff mass $\Lambda=3 \mathrm{GeV}$, the momentum carried by charm in this $\delta$ function approximation model is $P_{c}=1.66 \%$, which is slightly greater than in the MBM confining or effective mass models.

In Fig. 10 we compare the $c$ and $\bar{c}$ distributions in the MBM obtained using the confining model PDFs in the charmed hadrons with those computed from the effective mass model 
and $\delta$ function approximations, with a common cutoff mass $\Lambda=(3.0 \pm 0.2) \mathrm{GeV}$. The MBM confining model distributions are generally softer than those in the effective mass and $\delta$ function models, with the confining model giving a slightly broader shape, and the $\delta$ function model having the narrowest distribution. Within the uncertainty bands of the parameters (for clarity we only shown the uncertainty band for the confining model), the distributions are compatible with each other. In all three models the anticharm distributions are clearly harder than the charm, so that the qualitative features of the ratio $R_{c}$ in Fig. 9 are largely retained. Interestingly, the $\delta$ function model gives an $x$ dependence for the charm PDFs that closely resembled the shape of the effective mass model distributions for $\Lambda=3 \mathrm{GeV}$. This feature may be exploited in simplified calculations that seek only approximate feature of nonperturbative charm distributions.

A similar model to the MBM was constructed by Pumplin [21], based on the couplings of scalar mesons and baryons. The dominant meson-baryon contribution was assumed to be from the $\bar{D}^{0} \Lambda_{c}^{+}$state, and described by Eq. (3) with $N=2$ and an exponential form factor (4) with $\Lambda_{p}=4 \mathrm{GeV}$. The $c$ PDF in the $\Lambda_{c}$ was taken from Eq. (3) with $N=3$ and a dipole form factor with $\Lambda_{\Lambda_{c}}=2 \mathrm{GeV}$, while the $\bar{c}$ PDF in $\bar{D}$ was obtained assuming $N=2$ and a dipole form factor with $\Lambda_{D}=2 \mathrm{GeV}$. The magnitude of the resulting charm distributions in the nucleon was normalized so that the momentum carried by charm and anticharm quarks was identical, with their sum equal to the momentum carried by charm in the BHPS model with $1 \%$ probability, $P_{c}=0.57 \%$. A feature of this model is that the requirement that the momentum carried by $c$ and $\bar{c}$ quarks to be identical implies a nonzero net charm in the proton, $C^{(0)}-\bar{C}^{(0)} \neq 0$.

The resulting charm distribution in the nucleon in the scalar meson cloud model [21] is found to be similar in shape to that in the MBM with the confining vertex function, as Fig. 11 illustrates. The distribution is also very similar to that in the simplified BHPS fivequark model [2] discussed in Sec. II A (which is to be expected perhaps given that the scalar model is normalized to the charm in the BHPS model). The $\bar{c}$ distribution in the scalar model [21] is again harder than the $c$, while in the BHPS model the charm and anticharm distributions are assumed identical. Compared with the distributions in the confining MBM, the results for the lower limit of the form factor cutoff $\Lambda=2.8 \mathrm{GeV}$, are rather similar to the results of the other models shown in Fig. 11. However, for the central value $\Lambda=3 \mathrm{GeV}$ the MBM model gives a substantially larger intrinsic charm content. A similar result holds 

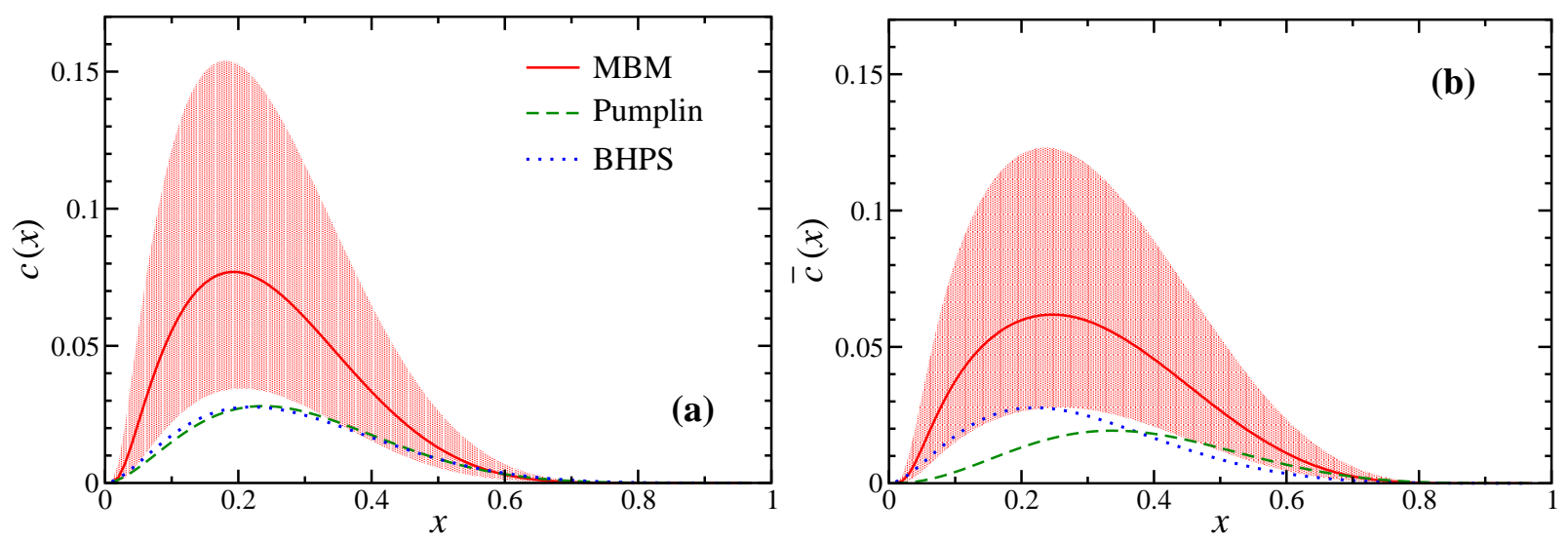

FIG. 11: (color online) Model dependence of the $(\mathbf{a}) c(x)$ and $(\mathbf{b}) \bar{c}(x)$ distributions in the nucleon, for the MBM with the confining model for the PDFs in charmed hadrons with cutoff mass $\Lambda=$ $(3.0 \pm 0.2) \mathrm{GeV}$ (red solid and shaded band), the Pumplin scalar meson cloud model (green dashed), and the BHPS intrinsic charm model (blue dotted).

for the anticharm distributions, except that the MBM results with confining form factor are slightly harder than the BHPS results.

Having explored the model dependence of the total intrinsic $c$ and $\bar{c}$ distributions in the nucleon, we can now directly confront the results with measurements of the charm structure function, $F_{2}^{c}$. This will provide additional constraints on the model parameters, complementing those of the inclusive $\Lambda_{c}$ production in $p p$ scattering discussed in Sec. IV A.

\section{Charm structure function}

The calculations of intrinsic charm in this analysis are normalized to inclusive charm production data in $p p$ collisions, as discussed in Sec. IV A. The results can also be confronted with data on the charm structure function $F_{2}^{c}$ obtained from measurements of charm production cross sections in deep-inelastic lepton scattering. In comparing with experimental measurements of $F_{2}^{c}$, in addition to intrinsic charm arising from nonperturbative fluctuations of the nucleon into states with 5 or more quarks, one must also consider the "extrinsic" charm arising from gluon radiation to $c \bar{c}$ pairs, which is described by perturbative QCD evolution.

To lowest order in the strong coupling constant $\alpha_{s}$, the charm structure function is 
straightforwardly related to the $c$ and $\bar{c}$ parton distributions in the nucleon,

$$
F_{2}^{c}\left(x, Q^{2}\right)=\frac{4 x}{9}\left[c\left(x, Q^{2}\right)+\bar{c}\left(x, Q^{2}\right)\right]
$$

When combining the two charm contributions, it is necessary to assign a scale $Q_{0}^{2}$ at which the nonperturbative charm is generated, and then evolve this, together with the perturbative component, to a common $Q^{2}$ for comparison with experiment. While the absolute scale of the intrinsic contribution is a characteristic ingredient of the model (in our case, the MBM), it is customary to set this to the effective charm quark mass, $Q_{0}^{2}=m_{c}^{2}=1.69 \mathrm{GeV}^{2}$.

The calculated charm structure function $F_{2}^{c}\left(x, Q^{2}\right)$ is shown in Fig. 12 for three different average $Q^{2}$ values ranging from $25 \mathrm{GeV}^{2}$ to $60 \mathrm{GeV}^{2}$, compared with data from the $\mathrm{H} 1$ [25] and ZEUS [26] Collaborations at HERA and with higher- $x$ data from EMC [24]. The extrinsic charm distribution is obtained from the CTEQ6.5 parametrization [62], where it is generated perturbatively through QCD evolution. The nonperturbative contribution to $F_{2}^{c}$ is computed from Eq. (48) using the MBM at the scale $Q_{0}^{2}$ and evolved to higher $Q^{2}$ using the next-to-leading order evolution code from Ref. [63]. Since the experimental $Q^{2}$ values are large compared to $m_{c}^{2}$, standard massless QCD evolution, in the form of the Variable Flavor Number Scheme, can be used.

At the lower two $Q^{2}$ values the extrinsic charm distributions give a reasonable fit to the data, both in the low- $x$ and high- $x$ regions, although at $Q^{2}=60 \mathrm{GeV}^{2}$ the perturbative results generally underestimate the EMC data at high $x$. The addition of the nonperturbative contribution raises the total $F_{2}^{c}$, depending on the amount of intrinsic charm assumed in the model. For the MBM with the confining model vertex functions, using the cutoff $\Lambda=(3.0 \pm 0.2) \mathrm{GeV}$ obtained from the fit to the inclusive $\Lambda_{c}^{+}$production data in $p p$ scattering generally overestimates the EMC $F_{2}^{c}$ data at large $x$ at the lowest $Q^{2}$ points. At the highest $Q^{2}$ value the calculated structure function is marginally consistent with the data at the lower edge of the error band. Using instead the MBM with the effective mass model vertex functions and the same cutoff $\Lambda=3.0 \mathrm{GeV}$, the peak at large $x$ is more pronounced, and thus overestimates the EMC data more somewhat more. Lowering the cutoff to $\Lambda=2.5 \mathrm{GeV}$ for the confining model, the resulting $F_{2}^{c}$ is in better agreement with the data, producing a smaller overestimate of the lower- $Q^{2}$ data points and resulting in a better fit to the $Q^{2}=60 \mathrm{GeV}^{2}$ data. Note that for such a small cutoff the average charm normalization $\langle n\rangle_{M B}^{(\text {charm })} \lesssim 0.5 \%$, which would significantly underestimate the inclusive $\Lambda_{c}^{+}$ 

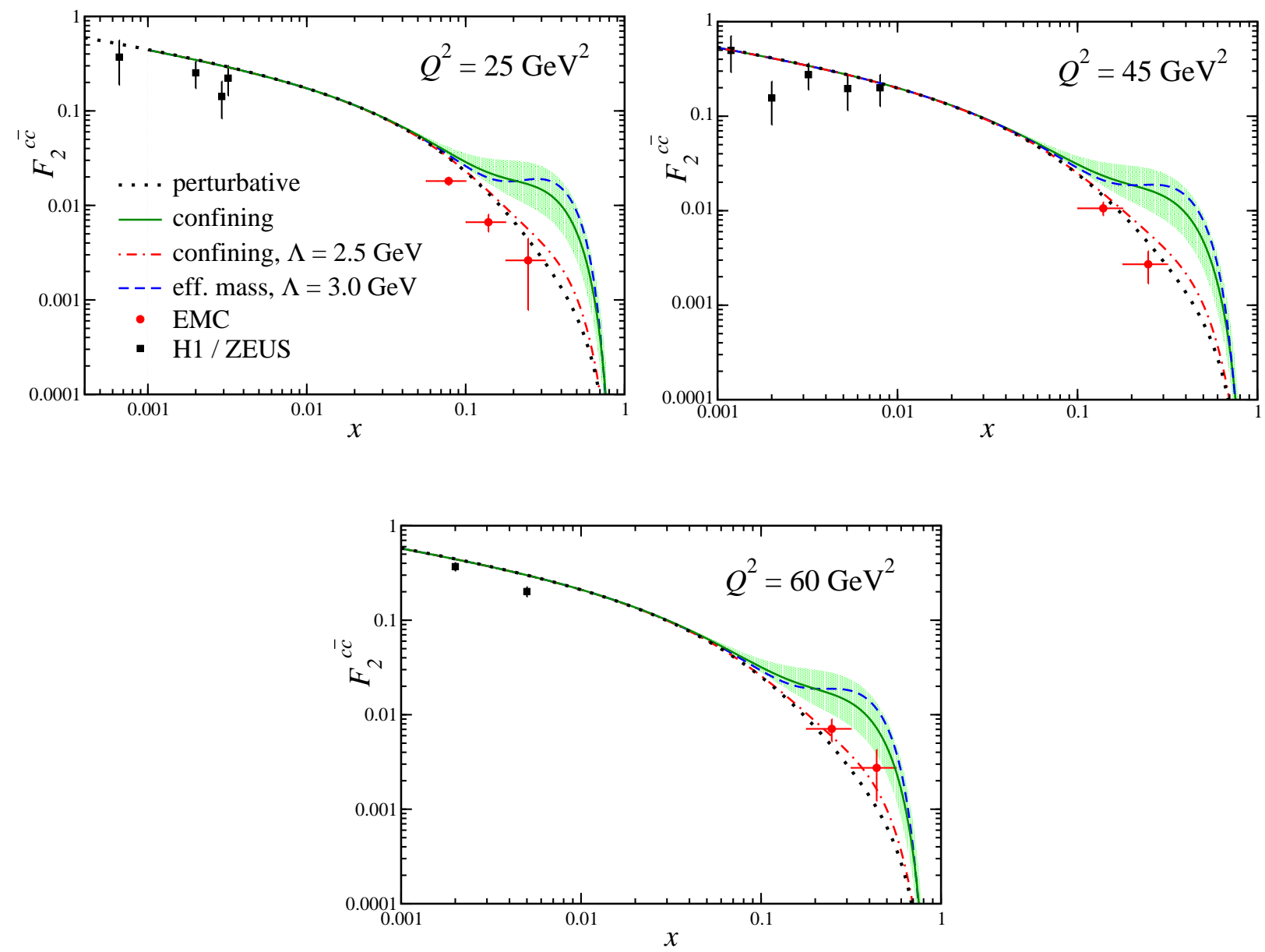

FIG. 12: (color online) Charm structure function $F_{2}^{c}$ at $Q^{2}=25 \mathrm{GeV}^{2}$ (top), $45 \mathrm{GeV}^{2}$ (middle), and $60 \mathrm{GeV}^{2}$ (bottom). The perturbative QCD calculation (black dotted line) is compared with nonperturbative charm contributions in the MBM using the confining model with cutoff $\Lambda=$ $(3.0 \pm 0.2) \mathrm{GeV}$ (green solid line and shaded band), confining model with $\Lambda=2.5 \mathrm{GeV}$ (red dotdashed line), and effective mass model with $\Lambda=3.0 \mathrm{GeV}$ (blue dashed line). The data are from H1 and ZEUS (black squares) and EMC (red circles).

production data (see Fig. 2).

In an earlier study [22], Pumplin, Lai and Tung carried out a global fit to high-energy data with phenomenological PDFs including an intrinsic charm term at the starting scale $Q_{0}=m_{c}$. Using several different phenomenological forms for the intrinsic charm (including the BHPS model, Eq. (2), the scalar MBM discussed in Sec. VIB, and a "sea-like" charm model), the magnitude of the charm contribution was varied until a substantial increase in the $\chi^{2}$ was found with the set of global high-energy data. The analysis found that the global 
fits could accommodate charm momentum fractions of $P_{c} \approx 2 \%$ in the BHPS and scalar MBM models and $P_{c} \approx 2.5 \%$ in the sea-like model at the $90 \%$ confidence level, which are significantly larger than the constraints from the EMC $F_{2}^{c}$ data, and at the upper boundary of the range allowed by the ISR R608 $\Lambda_{c}^{+}$production data. The more recent update [23] that includes NNLO corrections finds $P_{c} \leq 1.5 \%$ for the sea-like model and $P_{c} \leq 2.5 \%$ for the BHPS model at the scale $Q_{0}$.

We should note, however, that while the analysis in Ref. [22] fitted the precision low- $x$ charm structure function data from $\mathrm{H} 1$ and ZEUS, it did not include the EMC $F_{2}^{c}$ data at large $x$, which it is likely would have resulted in tighter constraints on the magnitude of the intrinsic charm. In a future study [64], we plan to perform a dedicated global analysis of all high-energy data, including the EMC measurements of $F_{2}^{c}$, to determine the constraints on the magnitude and shape of the nonperturbative charm component of the nucleon. Additional data on $F_{2}^{c}$ at large $x$ would of course be very valuable in providing additional information on this question.

\section{CONCLUSION}

In this work we have presented a comprehensive analysis of intrinsic charm in the nucleon using a phenomenological model formulated in terms of effective meson-baryon degrees of freedom, with couplings taken from $D N$ and $\bar{D} N$ scattering studies [38-40]. Within the MBM framework, the $c$ and $\bar{c}$ distributions in the nucleon are expressed as convolutions of $N \rightarrow$ charmed meson + baryon splitting functions and charm PDFs in the mesons and baryons. We have included in the calculation all of the low-lying pseudoscalar $D$ and vector $D^{*}$ mesons, together with the spin-1/2 $\Lambda_{c}, \Sigma_{c}$ and spin-3/2 $\Sigma_{c}^{*}$ baryons that couple to the proton, in contrast to some previous analyses that neglected the spin structure [21] and assumed dominance by the lowest mass $\bar{D}^{0} \Lambda_{c}^{+}$state $[15,17]$.

The splitting functions are determined essentially in terms of a single parameter, the momentum cutoff that regulates the ultraviolet behavior of the nucleon-meson-baryon form factor. The form factor is constrained by fitting to data on inclusive $\Lambda_{c}^{+}$production cross sections in $p p$ collisions from the ISR [41], which gives an exponential cutoff mass of $\Lambda=$ $(3.0 \pm 0.2) \mathrm{GeV}$. The resulting distributions are also consistent with the more recent SELEX data [42] on $\Lambda_{c}^{+} / \bar{\Lambda}_{c}^{-}$asymmetries, although here there is additional sensitivity to the $\bar{\Lambda}_{c}^{-}$ 
production mechanism.

The distributions of $c$ and $\bar{c}$ quarks in the charmed baryons and mesons are computed from a relativistic quark-spectator model, in which the momentum distributions of the quarks are parametrized through phenomenological quark-spectator-nucleon vertex functions. The resulting convolution integrals contain poles in the quark propagators, and we examined two methods for avoiding these poles. The first method involves using an effective charm mass that is sufficiently large that no poles occur in the physical region, while the second involves a vertex function that cancels the propagators in the denominator, thereby simulating the dynamics of quark confinement. For comparison we also considered a simplified model in which the charm distributions are approximated by $\delta$ functions at the fraction of the hadron mass carried by the constituent charm or anticharm quark. The various methods for avoiding propagator poles give rise to somewhat disparate distributions in hadrons, although the differences in the resulting intrinsic charm distributions in the nucleon are mitigated by the smearing effects of the convolution.

For values of the cutoff parameter favored by the inclusive $\Lambda_{c}^{+}$production data, we find that intrinsic charm is in fact dominated by the vector meson plus spin- $1 / 2$ baryon state $\bar{D}^{* 0} \Lambda_{c}^{+}$state, which is responsible for almost $70 \%$ of the total intrinsic charm content. The reasons for the singular strength of this channel are the very strong tensor coupling to vector mesons (reminiscent of the large tensor coupling of $\rho$ mesons in $N N$ interactions), and the additional momentum dependence introduced by the derivative interaction.

One of the unique characteristics of the MBM is the almost unavoidable asymmetry in the $x$ dependence of the resulting $c$ and $\bar{c}$ distributions in the nucleon, which reflects the different environments in which the charm and anticharm quarks exist (charmed baryon for $c, D$ and $D^{*}$ mesons for $\left.\bar{c}\right)$. Although the detailed shapes and absolute values of the $c$ and $\bar{c}$ PDFs depend on the models for the hadronic form factors and quark-spectator vertex functions, a universal feature of the MBM framework is the significantly harder $\bar{c}$ distribution compared with the $c$. The magnitude of the corresponding asymmetry ratio exceeds $50 \%$ at $x \gtrsim 0.5$, a result which is largely independent of the model parameters and scale. Comparison with the only available data on the charm structure function $F_{2}^{c}$ at large values of $x$ from the EMC [24], where the intrinsic charm is predicted to be most significant $(x \gtrsim 0.1)$, places tighter limits on the size of the momentum cutoff $\Lambda$ in the MBM. This suggests that the amount of intrinsic charm needed to describe the inclusive $p p$ data would lead to an overestimate of the 
DIS charm cross sections, although the $F_{2}^{c}$ data themselves are somewhat inconclusive, with some data points indicating an excess over the perturbative QCD expectations and others consistent with no intrinsic charm at all. At the very least, the large- $x F_{2}^{c}$ results illustrate the potential value of such data, and it is hoped that future measurements, such as at the proposed Electron-Ion Collider [34-36], will clarify the situation.

Other possibilities for identifying intrinsic charm experimentally include the measurement of $W$ and $Z$ cross sections at the LHC [65], where in the case of $W$ production, up to $1 / 3$ of the cross sections at $\sqrt{s}=7 \mathrm{TeV}$ and as much as $40 \%$ at $\sqrt{s}=14 \mathrm{TeV}$ can arise through charm production. However, although charm plays a significant role in these reactions, the dominant charm contributions occur at low rapidity where perturbative charm is expected to dominate. Alternatively, photon plus charm jet production at LHC energies, and in particular the transverse momentum distribution of prompt photons, has been identified [66] as potentially sensitive to intrinsic charm.

Another promising set of observables to study is charge asymmetries in the production of charmed hadrons such as $D^{+} / D^{-}, D_{s}^{+} / D_{s}^{-}$or $\Lambda_{c} / \bar{\Lambda}_{c}$ using proton, pion or $\Sigma^{-}$beams, as well as virtual photon probes in deep-inelastic lepton scattering. Particularly useful are comparisons of cross sections involving different incident beams, which in principle are sensitive to the so-called leading-particle effect [13], which involves a strong correlation between the quantum numbers of the produced charmed hadron and the beam hadron(s). For instance, in $\pi^{-} p \rightarrow D X$ scattering there is a preference to produce $D^{-}$and $D^{0}$ over $D^{+}$ and $\bar{D}^{0}$, which is attributable to the fact that the valence $(d \bar{u})$ content of the $\pi^{-}$appears also in the valence structure of $D^{-}$and $D^{0}$, but not in $D^{+}$and $\bar{D}^{0}$. Although significant charge asymmetries are observed in some cases, a number of possible mechanisms exist for such asymmetries (such as recombination of a produced charm quark with a valence quark or diquark from the beam, or coalescence between a charm and valence quark originating in the beam $[2,12])$, and it will be necessary to disentangle these processes from any preexisting charm in the initial state. However, any confirmation of an asymmetry between $c$ and $\bar{c}$ distributions in the nucleon at large $x$ would provide an unambiguous signature of intrinsic charm, beyond anything that could be generated from perturbative QCD.

Finally, we should mention some of the limitations of the MBM framework that has been used to compute the nonperturbative charm in this analysis. Unlike the fluctuations of the nucleon to baryons and light pseudoscalar mesons, such as pions, which are grounded in 
the chiral symmetry properties of QCD, the dissociation into the much heavier charmed mesons and baryons is a rather more model-dependent postulate. A reflection of this is the ad hoc restriction of the Fock state expansion in Eq. (6) to a particular set of meson-baryon states $M B$. While most analyses of charm in the MBM have simply assumed dominance by the lowest-mass $\bar{D}^{0} \Lambda_{c}^{+}$state, in this work we have attempted a more systematic treatment, including all low-lying states with the appropriate quantum numbers. As a guide, we have kept a close analogy with earlier $D N$ scattering analyses [38-40] which have provided constraints on the nucleon-meson-baryon couplings. Notwithstanding, in the absence of data on transitions to individual charmed meson and baryon states, it has been necessary to apply a universal cutoff parameter for all hadronic form factors, as well as construct phenomenological models for the charm quark distributions in the charmed hadrons. Further development of this approach will benefit from a combined analysis of all data, using both hadronic and leptonic probes. The future availability of inclusive charmed baryon production data as well as measurements of the charm structure function at large $x$ will provide vital benchmarks for establishing the presence of intrinsic charm in the nucleon.

\section{Acknowledgments}

We are grateful to J. Haidenbauer for helpful discussions about the Jülich meson-baryon scattering models. We also thank P. Jimenez-Delgado, D. Murdock, F. Steffens and R. Vogt for helpful discussions, and S. Kumano for his QCD evolution programs. TJH and JTL were supported in part by the US National Science Foundation under grant NSF-PHY-1205019. The work of TJH was also supported in part by DOE grant DE-FG02-87ER40365. WM was supported by the DOE contract No. DE-AC05-06OR23177, under which Jefferson Science Associates, LLC operates Jefferson Lab.

\section{Appendix A: Derivation of meson-baryon splitting functions}

In this appendix we provide additional technical details of the derivations of the splitting functions for the dissociation of a nucleon with four-momentum $P$ into a meson $M$ with momentum $k$ and baryon $B$ with momentum $p$. We consider dissociations into the SU(4) octet isoscalar $\Lambda_{c}$ and isovector $\Sigma_{c}$ baryons, and the decuplet $\Sigma_{c}^{*}$ baryon, accompanied by 
the charmed pseudoscalar $D$ and vector $D^{*}$ mesons. The transitions to specific isospin states are obtained using appropriate isospin transition factors, as discussed in Sec. IV.

The contribution of a specific meson-baryon component to the nucleon hadronic tensor $W_{\mu \nu}^{N}$ is defined in terms of the contributions $\delta^{[M B]} F_{1,2}^{N}$ to the structure functions as [54]

$$
\delta^{[M B]} W_{\mu \nu}^{N}(P, q)=\widetilde{g}_{\mu \nu} \delta^{[M B]} F_{1}^{N}+\frac{\widetilde{P}_{\mu} \widetilde{P}_{\nu}}{P \cdot q} \delta^{[M B]} F_{2}^{N}
$$

where $q$ is the four-momentum of the external electromagnetic field, and we define $\widetilde{g}_{\mu \nu}=$ $-g_{\mu \nu}+q_{\mu} q_{\nu} / q^{2}$, and $\widetilde{P}_{\mu}=P_{\mu}-P \cdot q q_{\mu} / q^{2}$. Evaluating the one-loop diagram for the scattering from the meson $M$ gives

$$
\delta^{[M B]} W_{\mu \nu}^{N}=\frac{g_{D B N}^{2}}{16 \pi^{2}} \int_{0}^{1} d y \int_{0}^{\infty} \frac{d k_{\perp}^{2}}{y(1-y)} \frac{|F(s)|^{2}}{\left(M^{2}-s\right)^{2}} N_{\mu \nu}^{M B},
$$

where $y$ is the longitudinal momentum fraction carried by the meson, $F(s)$ is the $M B N$ hadronic factor, with the invariant mass squared of the $M B$ system $s$ defined in Eq. (7). The tensor $N_{\mu \nu}^{M B}$ is computed from the spin trace of the appropriate meson and baryon propagators and vertices, with explicit forms given below.

Within the framework of time-ordered perturbation theory evaluated in the IMF kinematics $\left(P_{L} \rightarrow \infty\right)$, with intermediate state particles on their mass-shells but off their "energyshells", the standard decomposition for the momentum variables is [67]

$$
\begin{aligned}
P_{0} & =P_{L}+\frac{M^{2}}{2 P_{L}}+\mathcal{O}\left(\frac{1}{P_{L}^{2}}\right), \\
k_{0} & =|y| P_{L}+\frac{k_{\perp}^{2}+m_{M}^{2}}{2|y| P_{L}}+\mathcal{O}\left(\frac{1}{P_{L}^{2}}\right), \\
p_{0} & =|1-y| P_{L}+\frac{k_{\perp}^{2}+M_{B}^{2}}{2|1-y| P_{L}}+\mathcal{O}\left(\frac{1}{P_{L}^{2}}\right),
\end{aligned}
$$

for the energies, and

$$
\begin{aligned}
& \boldsymbol{k}=|y| \boldsymbol{P}+\boldsymbol{k}_{\perp}, \\
& \boldsymbol{p}=|1-y| \boldsymbol{P}-\boldsymbol{k}_{\perp},
\end{aligned}
$$

for the three-momenta, with $\boldsymbol{k}_{\perp} \cdot \boldsymbol{P}=0$. In the non-vanishing forward limit one has $y \in[0,1]$, such that $|1-y|=(1-y)$. The expression in Eq. (A2) are evaluated in terms of inner products $P \cdot k, P \cdot p$ and $P \cdot k$ computed from Eqs. (A3) and (A4), and the corrections to the structure functions $\delta^{[M B]} F_{1,2}^{N}$ obtained by equating the coefficients of the tensors in Eqs. (A1) 
and (A2). The corrections to the $c$ and $\bar{c}$ distributions in Eqs. (8) are then extracted from $\delta^{[M B]} F_{1,2}^{N}$ using parton model relations analogous to Eq. (48).

\section{$N \rightarrow D B$ splitting}

The dissociation of a nucleon to a spin-1/2 charmed baryon $B=\Lambda_{c}$ or $\Sigma_{c}$ and a pseudoscalar $D$ meson is derived from the effective hadronic Lagrangian [40]

$$
\mathcal{L}_{D B N}=i g \bar{\psi}_{N} \gamma_{5} \psi_{B} \phi_{D}+\text { h.c. }
$$

where $\psi_{N}$ and $\psi_{B}$ are the nucleon and charmed baryon fields, respectively, $\phi_{D}$ is the spin-0 $D$ meson field, and the coupling constant $g \rightarrow g_{D B N}$. Treating the propagation of the meson and baryon fields as appropriate for point particles, the trace factor $N_{\mu \nu}^{D B}$ can be written as

$$
\begin{aligned}
N_{\mu \nu}^{D B} & =\frac{1}{2} \operatorname{Tr}\left[i \gamma_{5}(\not P+M) i \gamma_{5} W_{\mu \nu}^{D}(k, q)\left(\not p+M_{B}\right)\right] \\
& =\left(2 P \cdot p-2 M M_{B}\right) \widetilde{g}_{\mu \nu} F_{1}^{D}+\ldots
\end{aligned}
$$

where $W_{D}^{\mu \nu}$ is the hadronic tensor for the $D$ meson, with a form similar to that in Eq. (A1), and $F_{1}^{D}$ is the corresponding structure function which depends on the $\bar{c}$ distribution in $D$. Equating the coefficients of $\widetilde{g}_{\mu \nu}$ in Eqs. (A1), (A2) and (A6) then yields the convolution expression in Eq. (8a) with the splitting function in Eq. (16). Performing the analogous calculation for the recoil process involving scattering from the baryon $B$ confirms the symmetry relation (10), which follows from the global charge and momentum conservation relations in Eqs. (13) and (14).

\section{$\underline{N \rightarrow D^{*} B \text { splitting }}$}

For the interaction between the nucleon and spin-1/2 baryon with a vector $D^{*}$ meson, the effective Lagrangian is given by [40]

$$
\mathcal{L}_{D^{*} B N}=g \bar{\psi}_{N} \gamma_{\mu} \psi_{B} \theta_{D^{*}}^{\mu}+\frac{f}{4 M} \bar{\psi}_{N} \sigma_{\mu \nu} \psi_{B} F_{D^{*}}^{\mu \nu}+\text { h.c. }
$$

where $\theta_{D^{*}}^{\mu}$ is the vector meson field, with field strength tensor $F_{D^{*}}^{\mu \nu}=\partial^{\mu} \theta_{D^{*}}^{\nu}-\partial^{\nu} \theta_{D^{*}}^{\mu}$, the tensor operator $\sigma_{\mu \nu}=(i / 2)\left[\gamma_{m} u, \gamma_{n} u\right]$, and the vector and tensor couplings are $g \rightarrow g_{D^{*} B N}$ and $f \rightarrow f_{D^{*} B N}$. In this case the trace factor $N_{\mu \nu}^{D^{*} B}$ is given by

$$
\begin{aligned}
N_{\mu \nu}^{D^{*} B}= & \frac{1}{2} \operatorname{Tr}\left[(\not P+M)\left(g \gamma^{\alpha}+\frac{f}{2 M}\left(\Delta^{\alpha}-\gamma^{\alpha} \not \Delta\right)\right)\left(\not p+M_{B}\right)\right. \\
& \left.\times\left(g \gamma^{\beta}-\frac{f}{2 M}\left(\Delta^{\beta}-\gamma^{\beta} \not \Delta\right)\right)\right] W_{\mu \nu \alpha \beta}^{D^{*}}(k, q) \\
= & \left(g^{2} G_{v}+\frac{g f}{M} G_{v t}+\frac{f^{2}}{M^{2}} G_{t}\right) \widetilde{g}_{\mu \nu} F_{1}^{D^{*}}+\ldots
\end{aligned}
$$


where $\Delta=P-p$, and the kinematical factors $G_{v}, G_{v t}$ and $G_{t}$ are given in Eqs. (20). The rank-4 tensor for the interacting $D^{*}$ meson can be expressed in the form [44]

$$
W_{\mu \nu \alpha \beta}^{D^{*}}(k, q)=\left(\widetilde{g}_{\mu \nu} F_{1}^{D^{*}}+\frac{\widetilde{k}_{\mu} \widetilde{k}_{\nu}}{m_{D^{*}}^{2}} F_{2}^{D^{*}}\right) \widetilde{g}_{\alpha \beta},
$$

with $F_{1,2}^{D^{*}}$ the corresponding vector meson structure functions.

$$
\underline{N \rightarrow D B^{*} \text { splitting }}
$$

The interaction of a nucleon with a spin-3/2 charmed baryon $B=\Sigma_{c}^{*}$ and a pseudoscalar meson is given by the Lagrangian [40]

$$
\mathcal{L}_{D B^{*} N}=\frac{f}{m_{D}}\left(\bar{\Psi}_{B^{*}}^{\mu} \psi_{N} \partial_{\mu} \phi_{D}+\bar{\psi}_{N} \Psi_{B^{*}}^{\mu} \partial_{\mu} \phi_{D}\right),
$$

where $\Psi_{B^{*}}^{\mu}$ is the Rarita-Schwinger spinor-vector field, and the coupling $f \rightarrow f_{D B^{*} N}$. From Eq. (A10) the trace factor tensor can be written

$$
\begin{aligned}
N_{\mu \nu}^{D B^{*}} & =\frac{1}{2} \operatorname{Tr}\left[(\not P+M) \Lambda_{B^{*}}^{\alpha \beta}(p) \Delta_{\alpha} \Delta_{\beta} W_{\mu \nu}^{D}(k, q)\right] \\
& =\frac{4}{3}\left(P \cdot p+M M_{B^{*}}\right)\left(\frac{(p \cdot \Delta)^{2}}{M_{B^{*}}^{2}}-\Delta^{2}\right) \widetilde{g}^{\mu \nu} F_{1}^{D}+\ldots
\end{aligned}
$$

where the energy projector for the spinor-vector is

$$
\Lambda_{B^{*}}^{\alpha \beta}(p)=\left(\not p+M_{B^{*}}\right)\left(-g^{\alpha \beta}+\frac{\gamma^{\alpha} \gamma^{\beta}}{3}+\frac{\gamma^{\alpha} p^{\beta}-\gamma^{\beta} p^{\alpha}}{3 M_{B^{*}}}+\frac{2 p^{\alpha} p^{\beta}}{3 M_{B^{*}}^{2}}\right) .
$$

The inner products in Eq. (A11) can once again be obtained from Eqs. (A3) and (A4) with $B \rightarrow B^{*}$, which leads directly to the splitting function in Eq. (21).

\section{$\underline{N \rightarrow D^{*} B^{*} \text { splitting }}$}

Finally, for the nucleon splitting to a spin-3/2 charmed baryon $B^{*}$ coupled to a vector meson $D^{*}$ the effective hadronic Lagrangian is given by [40]

$$
\mathcal{L}_{D^{*} B^{*} N}=\frac{f}{m_{D^{*}}} i\left(\bar{\Psi}_{B^{*} \nu} \gamma^{5} \gamma_{\mu} \psi_{N}-\bar{\psi}_{N} \gamma^{5} \gamma_{\mu} \Psi_{B^{*} \nu}\right) F_{D^{*}}^{\mu \nu},
$$

where $f \rightarrow f_{D^{*} B^{*} N}$. This yields the resulting trace tensor

$$
N_{\mu \nu}^{D^{*} B^{*}}=\frac{1}{2} \operatorname{Tr}\left[(\not P+M) \gamma^{5} \gamma^{\alpha} \Lambda_{B^{*}}^{\alpha^{\prime} \beta^{\prime}}(p) \gamma^{5} \gamma^{\beta}\right] \mathcal{G}_{\alpha \beta \alpha^{\prime} \beta^{\prime}} \widetilde{g}_{\mu \nu} F_{1}^{D^{*}}+\ldots
$$

where we define

$$
\mathcal{G}_{\alpha \beta \alpha^{\prime} \beta^{\prime}}=\Delta_{\alpha \beta} \widetilde{g}_{\alpha^{\prime} \beta^{\prime}}-\Delta_{\alpha \beta^{\prime}} \widetilde{g}_{\alpha^{\prime} \beta}-\Delta_{\alpha^{\prime} \beta} \widetilde{g}_{\alpha \beta^{\prime}}+\Delta_{\alpha^{\prime} \beta^{\prime}} \widetilde{g}_{\alpha \beta}
$$

and $\Delta_{\alpha \beta} \equiv \Delta_{\alpha} \Delta_{\beta}$, and the other expressions are defined above. Evaluating the trace in Eq. (A14) and equating coefficients of the $\widetilde{g}_{\mu \nu}$ terms then leads to the convolution relation with the splitting function in Eq. (22). 


\section{Appendix B: Charm content of charmed baryons and mesons}

This appendix details the derivations of the $c$ and $\bar{c}$ quark distributions in charmed baryons and mesons within the relativistic quark-spectator model introduced in Sec. V. For consistency with the calculation of the hadronic splitting functions in Appendix A, we proceed from effective quark-hadron Lagrangians using time-ordered perturbation theory in the IMF to compute the PDFs in terms of phenomenological vertex functions.

\section{$\underline{\bar{c} \text { in } D}$}

To model the distribution of a $\bar{c}$ quark in the pseudoscalar $D$ meson we consider the effective Lagrangian describing the coupling of the $D$ to the $\bar{c}$ and a light quark $q$,

$$
\mathcal{L}_{\bar{c} q D}=i g \bar{\psi}_{\bar{c}} \gamma_{5} \psi_{q} \phi_{D}+\text { h.c. }
$$

where $\psi_{q}$ and $\psi_{\bar{c}}$ are the quark $q$ and $\bar{c}$ fields, and the effective coupling constant is $g \rightarrow g_{\bar{c} q D}$. The contribution to the hadronic tensor of the $D$ meson from scattering off the $\bar{c}$ quark with a spectator light quark $q$ can be written in analogy with Eq. (A2) for the hadronic calculation,

$$
\bar{c}_{D}(z)=\frac{N_{D}}{16 \pi^{2}} \int_{0}^{\infty} \frac{d \hat{k}_{\perp}^{2}}{z(1-z)} \frac{|G(\hat{s})|^{2}}{\left(m_{D}^{2}-\hat{s}\right)^{2}} \widehat{T}^{\bar{c} q},
$$

where $\hat{k}_{\perp}$ denotes the internal quark transverse momentum in the $D$ meson and $z$ is the Bjorken scaling variable for the quark inside the $D$ meson, with the invariant mass squared $\hat{s}$ of the $\bar{c} q$ pair defined in Eq. (32). The normalization factor $N_{D}$ is determined from valence quark number conservation in the $D$ meson, Eq. (33).

The trace factor $\widehat{T}^{\bar{c} q}$ can be computed from the quark-level "handbag" diagram, yielding

$$
\begin{aligned}
\widehat{T}^{\bar{c} q} & =\frac{1}{4 \hat{k}^{+}} \operatorname{Tr}\left[i \gamma_{5}\left(\hat{\not h}+m_{\bar{c}}\right) \gamma^{+}\left(\hat{\not b}+m_{\bar{c}}\right)\left(-i \gamma_{5}\right)\left(-\hat{\not p}+m_{q}\right)\right], \\
& =2\left(\hat{p} \cdot \hat{k}+m_{\bar{c}} m_{q}\right),
\end{aligned}
$$

which follows from the on-mass-shell condition in time-ordered perturbation theory, $\hat{k}^{2}=m_{\bar{c}}^{2}$, with $\hat{p}$ the four-momentum of the spectator quark. The $\gamma^{+}$structure arises from reducing the hard scattering amplitude $\gamma^{\mu}\left(\hat{\not k}+q+m_{\bar{c}}\right) \gamma^{\nu} \delta\left((\hat{k}+q)^{2}-m_{\bar{c}}^{2}\right)$ to its leading twist approximation [68], $\gamma^{+} /\left(2 \hat{k}^{+}\right) \delta(1-z / \hat{y})$, where $\hat{y}$ is the parton fraction of the hadron momentum, after equating the coefficients of $g^{\mu \nu}$ and selecting the + component of the external photon current.

The result in Eq. (31) of Sec. V is then obtained by inserting the expression for $\widehat{T}^{\bar{c} q}$ in Eq. (B3) into Eq. (B2), and using the IMF momenta as in Eq. (A3) but with the replacements $y \rightarrow z, M \rightarrow M_{D}, m_{D} \rightarrow m_{\bar{c}}$, and $M_{B} \rightarrow m_{q}$. 
$\bar{c}$ in $D^{*}$

For the distribution in the $D^{*}$ meson, the following simple vector form is chosen for the Lagrangian describing the $\bar{c} q D^{*}$ interaction,

$$
\mathcal{L}_{\bar{c} q D^{*}}=g \bar{\psi}_{\bar{c}} \gamma_{\mu} \psi_{q} \theta_{D^{*}}^{\mu}+\text { h.c. }
$$

where $g \rightarrow g_{D^{*} \bar{q} q}$. This yields the trace factor

$$
\begin{aligned}
\widehat{T}^{(\bar{c} q)^{*}} & =\frac{1}{4 \hat{k}^{+}} \operatorname{Tr}\left[\left(-\hat{\not p}+m_{q}\right) \gamma^{\alpha}\left(\hat{\not h}+m_{\bar{c}}\right) \gamma^{+}\left(\hat{\not b}+m_{\bar{c}}\right) \gamma^{\beta}\right] \widetilde{g}_{\alpha \beta} \\
& =4\left(\frac{(P \cdot \hat{p})(P \cdot \hat{k})}{m_{D^{*}}^{2}}+\frac{3}{2} m_{\bar{c}} m_{q}+\frac{\hat{p} \cdot \hat{k}}{2}\right) .
\end{aligned}
$$

Applying the same procedure as for the $\bar{c}$ distribution inside the pseudoscalar $D$ meson, one immediately arrives at Eq. (37).

c in $\Lambda_{c}, \Sigma_{c}$

The charm quark distributions inside charmed baryons are obtained from an expression analogous to that in Eq. (B2),

$$
c_{B}(z)=\frac{N_{B}}{16 \pi^{2}} \int_{0}^{\infty} \frac{d \hat{k}_{\perp}^{2}}{z(1-z)} \frac{|G(\hat{s})|^{2}}{\left(m_{B}^{2}-\hat{s}\right)^{2}} \widehat{T}^{c[q q]},
$$

where $\widehat{T}^{c[q q]}$ is the corresponding trace factor for the scattering from the $c$ quark with a spectator diquark $[q q]$ in the intermediate state. For spin-1/2 baryons, we consider the scalar interaction between the $c$ and $[q q]$ quarks and the charmed baryon, given by the Lagrangian

$$
\mathcal{L}_{c[q q] B}=g \bar{\psi}_{B} \psi_{c} \phi_{[q q]}+\text { h.c. }
$$

with $g \rightarrow g_{c[q q] B}$, and $\phi_{[q q]}$ is the field of the scalar diquark. The trace factor $\widehat{T}^{c[q q]}$ can be explicitly derived as

$$
\begin{aligned}
\widehat{T}^{c[q q]} & =\frac{1}{4 \hat{k}^{+}} \operatorname{Tr}\left[\left(\not P+M_{B}\right)\left(\hat{\not b}+m_{c}\right) \gamma^{+}\left(\hat{\not b}+m_{c}\right)\right] \\
& =2\left(P \cdot \hat{k}+m_{c} M_{B}\right),
\end{aligned}
$$

giving the net result for the charm quark distribution inside a spin-1/2 baryon in Eq. (38). c in $\Sigma_{c}^{*}$

For the charm density of the spin-3/2 $B^{*}$ baryons the following Lagrangian is adopted,

$$
\mathcal{L}_{c[q q]^{*} B^{*}}=\frac{g}{m_{[q q]^{*}}} i\left(\bar{\Psi}_{B^{*} \nu} \gamma_{\mu} \psi_{c}-\bar{\psi}_{c} \gamma_{\mu} \Psi_{B^{*} \nu}\right) F_{[q q]^{*}}^{\mu \nu},
$$


with $g \rightarrow g_{c[q q]^{*} B^{*}}$, which correctly gives the parities of the physical $B^{*}$ and quark fields. Again, the field strength tensor here has the form $F_{[q q]^{*}}^{\mu \nu}=\partial^{\mu} \theta_{[q q]^{*}}^{\nu}-\partial^{\nu} \theta_{[q]^{*}}^{\mu}$, where $\theta_{[q q]^{*}}$ denotes the (spin-1) axial-vector diquark. The trace factor in this case is found to be

$$
\widehat{T}^{c[q q]^{*}}=\frac{1}{4 m_{[q q]^{*}}^{2} \hat{k}^{+}} \operatorname{Tr}\left[\Lambda_{B^{*}}^{\beta^{\prime} \alpha^{\prime}}(P) \gamma^{\alpha}\left(\hat{\not b}+m_{c}\right) \gamma^{+}\left(\hat{\not b}+m_{c}\right) \gamma^{\beta}\right] \mathcal{G}_{\alpha \beta \alpha^{\prime} \beta^{\prime}}
$$

After re-indexing, $\mathcal{G}_{\alpha \beta \alpha^{\prime} \beta^{\prime}}$ is given by Eq. (A14), the exchange boson carries the fourmomentum $\hat{\Delta}=P-\hat{k}$, and the metric tensor of the massive, spin-1 diquark is $\widetilde{g}^{\mu \nu}=$ $-g^{\mu \nu}+P^{\mu} P^{\nu} / m_{[q]^{*}}^{2}$. Computing the trace and contractions of Eq. (B10) and evaluating the result with the appropriate kinematic definitions analogous to Eq. (A3), one may obtain Eq. (41) as given in Sec. V B.

\section{Appendix C: Phenomenological Fit Parameters}

Following Ref. [21], we provide here simple three-parameter fits to the $c$ and $\bar{c}$ PDFs in the MBM computed using several different models for the charm quark distributions in the charmed mesons and baryons, including the confining model, effective mass model, and the $\delta$ function model. The parametric form for the charm distributions in the nucleon for the confining and effective mass models is taken to be

$$
\begin{aligned}
& c(x)=C^{(0)} A x^{\alpha}(1-x)^{\beta}, \\
& \bar{c}(x)=C^{(0)} \bar{A} x^{\bar{\alpha}}(1-x)^{\bar{\beta}},
\end{aligned}
$$

where the normalization constants $A=1 / B(\alpha+1, \beta+1)$ and $\bar{A}=1 / B(\bar{\alpha}+1, \bar{\beta}+1)$, with $B$ the Euler beta function, ensure that the distributions are normalized to $C^{(0)}$.

For the $\delta$ function model, where the $c$ and $\bar{c}$ PDFs in the charmed hadrons are given by $\delta$ functions in $x$, it is more convenient to parametrize the distributions in Eqs. (46) as

$$
\begin{aligned}
& c(x)=C^{(0)} A x^{\alpha}\left(x_{B}-x\right)^{\beta} \theta\left(x_{B}-x\right), \\
& \bar{c}(x)=C^{(0)} \bar{A} x^{\bar{\alpha}}\left(x_{M}-x\right)^{\bar{\beta}} \theta\left(x_{M}-x\right),
\end{aligned}
$$

with $x_{B}$ and $x_{M}$ given by Eq. (47).

For convenience, we give the parameters for the PDFs normalized to $C^{(0)}$, which can be varied within each model according to preference. Although the actual normalization $C^{(0)}$ in the MBM is modified through the form factor cutoff $\Lambda$, which affects also the shape of 
TABLE III: Best fit parameter values for the $c$ and $\bar{c}$ distributions in the nucleon in Eqs. (C1) and (C2) in the MBM for a central cutoff mass $\Lambda=3.0 \mathrm{GeV}$.

\begin{tabular}{c|ccc}
\hline \hline $\begin{array}{c}c, \bar{c} \text { fit } \\
\text { parameters }\end{array}$ & $\begin{array}{c}\text { confining } \\
\text { model }\end{array}$ & $\begin{array}{c}\text { effective } \\
\text { mass model }\end{array}$ & $\begin{array}{c}\delta \text { function } \\
\text { model }\end{array}$ \\
\hline$A$ & $1.720 \times 10^{2}$ & $1.052 \times 10^{2}$ & $2.638 \times 10^{5}$ \\
$\alpha$ & 1.590 & 3.673 & 4.266 \\
$\beta$ & 6.586 & 10.16 & 4.485 \\
\hline $\bar{A}$ & $7.404 \times 10^{1}$ & $4.160 \times 10^{0}$ & $2.463 \times 10^{4}$ \\
$\bar{\alpha}$ & 1.479 & 4.153 & 5.003 \\
$\bar{\beta}$ & 4.624 & 6.800 & 4.857 \\
\hline
\end{tabular}

the resulting distribution, in practice the change in the shape parameters over the range $2.8 \lesssim \Lambda \lesssim 3.2 \mathrm{GeV}$ is small. The parameters in Table III are given for the central fit value $\Lambda=3.0 \mathrm{GeV}$, determined from the inclusive $\Lambda_{c}^{+}$production data in $p p$ scattering, for which the normalization is $C^{(0)}=2.4 \%$. The parametrization may be extended to smaller values of the cutoff, $\Lambda \approx 2.5 \mathrm{GeV}$, for qualitative comparisons, although the changes in shape begin to become more appreciable as one moves away from the above range.

[1] Heavy quark asymmetries can be generated perturbatively, but only at three-loop order, see S. Catani, D. de Florian, G. Rodrigo and W. Vogelsang, Phys. Rev. Lett. 93, 152003 (2004).

[2] S. J. Brodsky, P. Hoyer, C. Peterson and N. Sakai, Phys. Lett. B 93, 451 (1980).

[3] D. Drijard et al., Phys. Lett. B 81, 250 (1979).

[4] K. L. Giboni et al., Phys. Lett. B 85, 437 (1979).

[5] W. Lockman et al., Phys. Lett. B 85, 443 (1979).

[6] D. Drijard et al., Phys. Lett. B 85, 452 (1979).

[7] S. J. Brodsky, C. Peterson and N. Sakai, Phys. Rev. D 23, 2745 (1981).

[8] E. Hoffman and R. Moore, Z. Phys. C 20, 71 (1983).

[9] B. W. Harris, J. Smith and R. Vogt, Nucl. Phys. B461, 181 (1996).

[10] R. Vogt and S. J. Brodsky, Phys. Lett. B 349, 569 (1995).

[11] R. Vogt, Nucl. Phys. B446, 149 (1995). 
[12] R. Vogt and S. J. Brodsky, Nucl. Phys. B438, 261 (1995).

[13] R. Vogt and S. J. Brodsky, Nucl. Phys. B478, 311 (1996).

[14] G. Ingelman and M. Thunman, Z. Phys. C 73, 505 (1997).

[15] W. Melnitchouk and A. W. Thomas, Phys. Lett. B 414, 134 (1997).

[16] F. M. Steffens, W. Melnitchouk and A. W. Thomas, Eur. Phys. J. C 11, 673 (1999).

[17] F. S. Navarra, M. Nielsen, C. A. A. Nunes and M. Teixeira, Phys. Rev. D 54, 842 (1996).

[18] F. S. Navarra and M. Nielsen, Phys. Lett. B 443, 285 (1998).

[19] F. O. Durães, F. S. Navarra and M. Nielsen, Phys. Lett. B 498, 169 (2001).

[20] V. P. Goncalves, F. S. Navarra and T. Ullrich, Nucl. Phys. A 842, 59 (2010).

[21] J. Pumplin, Phys. Rev. D 73, 114015 (2006).

[22] J. Pumplin, H. L. Lai and W.-K. Tung, Phys. Rev. D 75, 054029 (2007).

[23] S. Dulat, T.-J. Hou, J. Gao, J. Huston, J. Pumplin, C. Schmidt, D. Stump and C. -P. Yuan, arXiv:1309.0025 [hep-ph].

[24] J. J. Aubert et al., Nucl. Phys. B213, 31 (1983); Phys. Lett. B 94, 96 (1980); ibid. B 110, 73 (1982).

[25] A. Aktas et al., Eur. Phys. J. C 45, 23 (2006).

[26] S. Chekanov et al., JHEP 0707, 074 (2007).

[27] A. D. Martin, W. J. Stirling, R. S. Thorne and G. Watt, Eur. Phys. J. C 63, 189 (2009).

[28] P. Jimenez-Delgado and E. Reya, Phys. Rev. D 79, 074023 (2009).

[29] S. Kumano, J. Phys. Conf. Ser. 312, 032005 (2011), and reference therein.

[30] J. Riedl, A. Schäfer and M. Stratmann, Eur. Phys. J. C 52, 987 (2007).

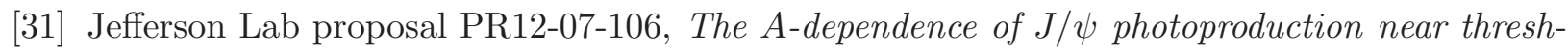
old, E. Chudakov et al., spokespersons.

[32] N. Brambilla et al., Eur. Phys. J. C 71, 1534 (2011).

[33] S. J. Brodsky, F. Fleuret, C. Hadjidakis and J. P. Lansberg, Phys. Rep. 522, 239 (2013).

[34] A. Deshpande et al., Ann. Rev. Nucl. Part. Sci. 55, 165 (2005).

[35] D. Boer et al., arXiv:1108.1713 [nucl-th].

[36] A. Accardi et al., arXiv:1212.1701 [nucl-ex].

[37] A. Sibirtsev, K. Tsushima and A. W. Thomas, Phys. Rev. C 63, 044906 (2001).

[38] J. Haidenbauer, G. Krein, U.-G. Meißner and A. Sibirtsev, Eur. Phys. J. A 33, 107 (2007).

[39] J. Haidenbauer, G. Krein, U.-G. Meißner and A. Sibirtsev, Eur. Phys. J. A 37, 55 (2008). 
[40] J. Haidenbauer, G. Krein, U.-G. Meißner and L. Tolos, Eur. Phys. J. A 47, 18 (2011).

[41] P. Chauvat et al., Phys. Lett. B 199, 304 (1987).

[42] F. G. Garcia et al., Phys. Lett. B 528, 49 (2002).

[43] V. R. Zoller, Z. Phys. C 53, 443 (1992).

[44] W. Melnitchouk and A. W. Thomas, Phys. Rev. D 47, 3794 (1993).

[45] H. Holtmann, A. Szczurek and J. Speth, Nucl. Phys. A596, 631 (1996).

[46] J. Speth and A. W. Thomas, Adv. Nucl. Phys. 24, 83 (1998).

[47] S. Kumano, Phys. Rep. 303, 183 (1998).

[48] W. Melnitchouk, A. W. Schreiber and A. W. Thomas, Phys. Rev. D 49, 1183 (1994).

[49] M. Burkardt, K. S. Hendricks, C. -R. Ji, W. Melnitchouk and A. W. Thomas, Phys. Rev. D 87, 056009 (2013).

[50] R. Machleidt, K. Holinde and C. Elster, Phys. Rep. 149, 1 (1987).

[51] M. Hoffman, J. W. Durso, K. Holinde, B. C. Pearce and J. Speth, Nucl. Phys. A593, 341 (1995).

[52] B. Holzenkamp, K. Holinde and J. Speth, Nucl. Phys. A500, 485 (1989).

[53] E. R. Cazaroto, V. P. Goncalves, F. S. Navarra and M. Nielsen, arXiv:1302.0035 [hep-ph].

[54] J. Beringer et al. [Particle Data Group], Phys. Rev. D 86, 010001 (2012).

[55] G. Hohler and E. Pietarinen, Nucl. Phys. B95, 210 (1975).

[56] J. S. Conway et al., Phys. Rev. D 39, 92 (1989).

[57] K. Wijesooriya, P. E. Reimer and R. J. Holt, Phys. Rev. C 72, 065203 (2005).

[58] W. Aitkenhead et al., Phys. Rev. Lett. 45, 157 (1980).

[59] A. I. Signal and A. W. Thomas, Phys. Lett. B 191, 205 (1987).

[60] W. Melnitchouk and M. Malheiro, Phys. Rev. C 55, 431 (1997); Phys. Lett. B 451, 224 (1999).

[61] F. E. Close and A. W. Thomas, Phys. Lett. B 212, 227 (1988).

[62] W.-K. Tung et al., JHEP 0702, 053 (2007).

[63] M. Miyama and S. Kumano, Comput. Phys. Commun. 94, 185 (1996).

[64] T. J. Hobbs, P. Jimenez-Delgado, J. T. Londergan and W. Melnitchouk, in preparation (2013).

[65] F. Halzen, Y. S. Jeong and C. S. Kim, arXiv:1304.0322.

[66] V. A. Bednyakov et al., arXiv:1305.3548.

[67] S. D. Drell, D. J. Levy and T. -M. Yan, Phys. Rev. D 1, 1035 (1970).

[68] P. J. Mulders, A. W. Schreiber and H. Meyer, Nucl. Phys. A 549, 498 (1992). 